Boletín de la Sociedad Geológica Mexicana

Volumen 64, NúM. 1, 2012, P. 91-108

\title{
Paleosuelos en secuencias coluvio-aluviales del Pleistoceno - Holoceno en Tlaxcala: registros paleoambientales del poblamiento temprano en el centro de México
}

\author{
Berenice Solís-Castillo ${ }^{1, *}$, Elizabeth Solleiro-Rebolledo ${ }^{2}$, Sergey Sedov², \\ Cesar Salcido-Berkovich ${ }^{1}$ \\ ${ }^{1}$ Posgrado en Ciencias de la Tierra, Instituto de Geología, UNAM. Ciudad Universitaria, 04510, México, D.F. \\ ${ }^{2}$ Instituto de Geología, UNAM. Ciudad Universitaria, 04510, México, D.F. \\ *bsolisc.geologia@gmail.com
}

\begin{abstract}
Resumen
El estudio de paleosuelos cobra importancia al considerar la posición que ocupan en el paisaje, ya que representan un continuo que cambia en función de la geoforma y constituyendo una toposecuencia. En este contexto, aquí se muestran los resultados de tres secciones paleopedológicas localizadas en el Bloque Tlaxcala y los efectos de la pedogénesis dependiendo de su posición en el paisaje, de las condiciones ambientales y del origen del material parental (coluvio-aluvial). Las secciones se localizan en la cima (sección Tlalpan C), en el talud (Concepción C) y en el fondo del valle (Huexoyucan) y cubren desde la Etapa Isotópica de Oxígeno 3 (EIO3), el Último Máximo Glacial (UMG), el Glacial tardío (GT), hasta el Holoceno temprano (HT); este último con énfasis en las condiciones ambientales del poblamiento temprano en México. Los procesos formadores de suelo se dedujeron a través del análisis de los rasgos morfológicos, granulométricos, susceptibilidad magnética, contenido paleomicrobotánico, los datos de $\delta^{13} \mathrm{C}$ y fechamientos. Los resultados muestran que los paleosuelos desarrollados durante la EIO3 son Luvisoles con rasgos vérticos, los cuales están presentes en las posiciones de mayor altitud, cambiando hacia Fluvisoles hísticos en la base de la toposecuencia, evidenciando un clima estacional (con oscilaciones entre condiciones frías-húmedas y secas). Durante el UMG los Luvisoles se presentan tanto en la cima como en la base, interpretándose como resultado de un clima húmedo y frío. En el GT se tienen suelos de bajo grado de desarrollo que contienen material retrabajado y redepositado, y que conforman Gleysoles en las diferentes posiciones del relieve, mostrando ambientes inestables. Los paleosuelos del HT se han clasificado como Luvisoles con propiedades estágnicas, propios de un clima húmedo y frío, pero bien drenados en las partes altas. En la prospección arqueológica del poblamiento temprano de la región, la unidad del HT ofrece facilitar la labor de encontrar materiales de interés.
\end{abstract}

Palabras clave: paleosuelos coluvio-aluviales, toposecuencia, Pleistoceno-Holoceno, Tlaxcala, poblamiento temprano.

\begin{abstract}
The study of paleosols becomes important when considering the position that they occupy in the landscape because they are a continuum changing in relation to the landform, constituting a toposequence. Here we present the results from three paleopedological sections located in the Tlaxcala Block, the effects of pedogenesis depending on their relief position and the kind of parent material (colluvio-alluvial). The studied sections are located at the higher positions (Tlalpan C), in the talus (Concepción C), and in the valley bottom (Huexoyucan), and cover from the Oxygen Isotope Stage 3 (OIS3), the Last Glacial Maximum (LGM), the Late Glacial (LT), to the early Holocene (EH), the last being important because of the early peopling of Mexico. The soil-forming processes were deduced through analysis of the morphological characteristics, grain size distribution, magnetic susceptibility, paleomicrobotanical content, the $\delta^{13} \mathrm{C}$ data, and the dating analysis. Results show that paleosols developed during the OIS3 are Luvisols with vertic features, present in
\end{abstract}


the higher altitudes, changing to histic Fluvisols at the base of the toposequence, evidencing a seasonal climate (oscillating between cold-humid and dry conditions). During the LGM, Luvisols are present at the top and base of the landscape, and are interpreted as the result of a humid and cool environment. The $L G$ soils are less developed, containing reworked and redeposited material, that form Gleysols at different relief positions, indicating unstable environments. Paleosols of the LH are Luvisol with stagnic properties, formed in a humid and cool climate, but well drained in the higher elevations. Paleosols of LH offer a good perspective for archaeological prospection of the early peopling sites.

Keywords: coluvio-alluvial paleosols, toposequence, Pleistocene-Holocene, Tlaxcala, early peopling.

\section{Introducción}

Existe un fuerte debate sobre las edades del poblamiento inicial de América, ya que aunque se reconoce que éste ocurrió alrededor de los 11500 años AP (Fiedel, 2006), se tienen reportes de ocupación muy temprana desde 20000 hasta 40000 años AP, particularmente en la Cueva de Babicora, Baja California, con edades de 38000 - > 47000 años AP (Fujita et al., 2006); Valsequillo, Puebla, con más de 40000 años AP (González et al., 2006); El Cedral, San Luis Potosí, hace 33000 años (Lorenzo y Mirambell, 1986a); Tlapacoya, Estado de México, hace 22000 años (Mirambell, 1978; Lorenzo y Mirambell, 1986b). Estos datos controversiales derivan tanto del número limitado de hallazgos como de su documentación arqueológica y estratigráfica, en muchos casos insuficiente (Kozlowski y Bandi, 1992).

En el centro de México, las evidencias más claras, con fechamientos directos en huesos humanos, se enmarcan en los 11000 años AP (González et al., 2003), es decir en la transición Pleistoceno-Holoceno, cuando los cambios en el ambiente fueron drásticos delimitando los espacios a paisajes con características singulares que posiblemente influyeron en el patrón de dispersión de los primeros americanos. Sin embargo, la documentación de la evidencia arqueológica de los sitios estudiados (Peñón, Tlapacoya, Texcal) no ha permitido caracterizar a las sociedades tempranas asentadas en el centro de México, por lo que se han propuesto nuevas perspectivas para el estudio del hombre temprano en la región.

De particular interés resulta el estudio de la relación entre el patrón de movilidad de las sociedades cazadorasrecolectoras y las características del eco (geo) sistema, ya que estos grupos humanos desarrollaron adaptaciones culturales y tecnológicas para enfrentarse a nuevos paisajes durante su migración hacia el continente Americano.

Recientes investigaciones plantean un enfoque geoarqueológico de la prehistoria para deducir los mecanismos de la relación entre el hombre temprano y su ambiente, ya que la configuración del entorno durante la transición Pleistoceno-Holoceno varió espacial y temporalmente. Tales cambios modificaron el ordenamiento social propiciando adaptaciones culturales para las actividades de subsistencia (caza, recolección y pesca).

La reconstrucción de las condiciones del entorno durante un periodo de ocupación, en este caso el Pleistoceno final, no sólo proporciona información sobre las características del ambiente bajo las cuales el hombre temprano llegó al Altiplano Central, sino que también establece la distribución a una escala espacial más reducida de los microambientes, los cuales posiblemente restringieron las actividades de apropiación a la disposición y variedad de recursos potencialmente disponibles y aprovechables por los primeros pobladores durante el Cuaternario tardío.

Es por ello que en este trabajo se realizó una reconstrucción paleoambiental por medio del estudio pedológico, especialmente enfocado a las propiedades que constituyen la memoria del suelo (Targulian y Goriachkin, 2004), vinculadas al efecto del factor relieve en la distribución y extensión de los suelos. A pesar de su limitada resolución temporal, los paleosuelos poseen una alta resolución espacial, por lo que son instrumentos valiosos para reconstruir el mosaico de ambientes en una región. Por lo tanto, el análisis de las propiedades de los paleosuelos brinda un reflejo tanto de las condiciones ambientales predominantes como su relación con la distribución de los micro-ambientes. Estos escenarios ambientales están vinculados con el patrón espacial de aprovechamiento del medio.

Particularmente, el trabajo se enfocó a las condiciones paleoambientales en el Pleistoceno tardío-Holoceno, en localidades del estado de Tlaxcala, en donde se tienen evidencias de ocupación humana intensiva (Heine, 2003; Borejsza et al., 2008, Borejsza y Frederick, 2010) y, por ende, representa un esfuerzo por vincular los sitios de ocupación con los paisajes del pasado. En estudios previos, Sedov y colaboradores (2009) realizaron una reconstrucción paleoambiental en localidades de Tlaxcala, cubriendo un lapso mayor (Pleistoceno medio-Holoceno). En este trabajo se retoman parcialmente los resultados correspondientes a la Unidad Gris, pero con un enfoque diferente, ya que aquí se contempla la reconstrucción por medio del estudio de una toposecuencia, que permite tener una visión espacial de las variaciones ambientales. 


\section{2. Área de estudio, materiales y métodos}

\section{1. Área de estudio}

El área de estudio se ubica en el Bloque Tlaxcala que forma parte de la cuenca Puebla-Tlaxcala, entre las coordenadas $19^{\circ} 27^{\prime} 41.3^{\prime \prime} \mathrm{N} ; 98^{\circ} 18^{\prime} 52.5^{\prime \prime} \mathrm{W}, 2580 \mathrm{msnm}$ y $19^{\circ} 22^{\prime} 16.7^{\prime \prime} \mathrm{N} ; 98^{\circ} 16^{\prime}$ 47.4” W, 2480 msnm (Figura 1). El Bloque Tlaxcala se elevó debido a una falla normal (LermoSamaniego y Bernal-Esquia, 2006) originada en el Mioceno Temprano (Mooser et al., 1996). El bloque se conforma de secuencias lacustres del Terciario las cuales fueron cubiertas por rocas volcánicas de conos monogenéticos del Cuaternario (Rico et al., 1997; Vilaclara et al., 2010). La parte superior está coronada por una secuencia de paleosuelos de naturaleza volcánica que cubre desde el Pleistoceno medio hasta el Reciente (Sedov et al., 2009).

Las condiciones ambientales en el área de estudio corresponden a un clima templado sub-húmedo. La temperatura media anual es de $13{ }^{\circ} \mathrm{C}$ con una precipitación anual de $838 \mathrm{~mm}$ (García, 1988). La vegetación está constituida por un bosque de encino con especies: Pinus oaxacana, Quercus crassipes, Quercus castanea, Quercus dentralis, Quercus obtuse y Arbustus glandosa, en áreas poco perturbadas (Klink, 1973). Los suelos de la región están caracterizados por Litosoles, Regosoles, Cambisoles, Xerosoles, Luvisoles, Andosoles y Antroposoles (Werner et al., 1978).

\subsection{Materiales y métodos}

Se estudiaron con detalle 3 secciones pedoestratigráficas, ubicadas en diferentes posiciones geomorfológicas (cima, talud, fondo del valle), con el fin de tener una toposecuencia, la cual permitiera hacer una reconstrucción de la distribución espacial y temporal de las unidades. Asimismo, se hizo una correlación con los resultados de Sedov y colaboradores (2009) que enriqueciera la reconstrucción paleoambiental.

La descripción detallada en campo de las características morfológicas de cada uno de los perfiles se realizó con base en los criterios de la World Reference Base for soil resources (WRB; IUSS, 2006) y los propuestos por Retallack (1990). El muestreo se llevó a cabo partiendo de la identificación de los paleosuelos y sus respectivos horizontes diagnósticos. Es de mencionar que los análisis de laboratorio se restringen a aquellas propiedades que se asocian con los procesos pedogenéticos y que pueden ser interpretados como registro paleoambiental.

Se hicieron láminas delgadas con muestras inalteradas que fueron impregnadas con resina Cristal MC- 40 para el análisis micromorfológico bajo el microscopio petrográfico; la identificación de los rasgos edafogenéticos se hizo de acuerdo con el manual de Bullock et al. (1985). La separación de las fracciones de arena limo y arcilla se hizo eliminando previamente materia orgánica con $\mathrm{H}_{2} \mathrm{O}_{2}$ al $15 \%$, y óxidos de hierro siguiendo la técnica de Mehra y Jackson (1960). La susceptibilidad magnética de baja frecuencia se evaluó con muestras de $8 \mathrm{~cm}^{3}$ de cada horizonte, por medio de un susceptibilímetro Bartington modelo MS2 y un sensor dual tipo MS2B del Laboratorio de Paleomagnetismo del Instituto de Geofísica, UNAM. La cantidad de carbono orgánico total se evaluó por medio de un analizador CHNS/O, Perkin Elmer 2400, serie II. Los valores de $\delta^{13} \mathrm{C}$ de los horizontes orgánicos se determinaron en el laboratorio de Espectrometría de Masas de Isótopos Estables del Instituto de Geología de la UNAM.

Dado que la conservación de polen en suelos es pobre, las extracciones polínicas se hicieron únicamente en los horizontes de turba (paleosuelo 6 de Huexoyucan), mediante la técnica palinológica estándar. La identificación de los palinomorfos se realizó en un microscopio Olympus BX50 reconociendo el número y tipo de aberturas, ornamentación, tamaño y forma del grano de polen, así como sus estructuras. En los mismos horizontes, se identificaron las especies de diatomeas de acuerdo con géneros ya establecidos en los manuales elaborados por Kramer y Lange-Bertalot (1991) y las muestras se prepararon con la técnica detallada por Patrick y Reimer $(1966,1975)$.

La edad de los paleosuelos se estableció con base en fechamientos de materia orgánica total de algunos horizontes tipo A, realizados en los laboratorios Beta Analytic, (Miami, Florida USA). Las fechas se reportan en edades calibradas de acuerdo con los datos enviados por el laboratorio, el cual usa la base de calibración INTCAL04 (Reimer et al., 2004). En los casos en que el laboratorio no efectuó la calibración por encontrarse fuera del rango, se usó el programa CalPal Online (CalPal Online, 2007). Cabe destacar que los fechamientos de los horizontes A representa la edad mínima, es decir, marca el periodo en el que finaliza el desarrollo del suelo y, por lo mismo, la pedogénesis pudo iniciarse con anterioridad.

\section{Resultados}

Las tres secciones pedoestratigráficas incluyen suelos modernos y paleosuelos sepultados que fueron estudiados en diferentes posiciones en el paisaje: la zona alta está definida por el perfil Tlalpan $\mathrm{C}$ que se localiza en las coordenadas geográficas $19^{\circ} 27^{\prime} 34.8^{\prime \prime} \mathrm{N}$; 98 $98^{\circ} 18^{\prime} 51.8^{\prime \prime} \mathrm{W}$, a $2558 \mathrm{msnm}$. La transición de la zona alta hacia el valle está caracterizada por la sección Concepción C se ubica en las coordenadas geográficas $19^{\circ} 27^{\prime} 28.4^{\prime \prime} \mathrm{N}$; 98 $98^{\circ} 19^{\prime}$ 2.4” W, a $2541 \mathrm{msnm}$. Finalmente, el valle se define por el perfil Huexoyucan que se localiza en las coordenadas geográficas $19^{\circ} 22^{\prime} 16.7^{\prime \prime} \mathrm{N}$; $98^{\circ} 16^{\prime} 47.4^{\prime \prime}$ W a 2480 msnm.

\subsection{Pedoestratigrafía de las secciones estudiadas}

Las secciones Tlalpan C, Concepción C y Huexoyucan 


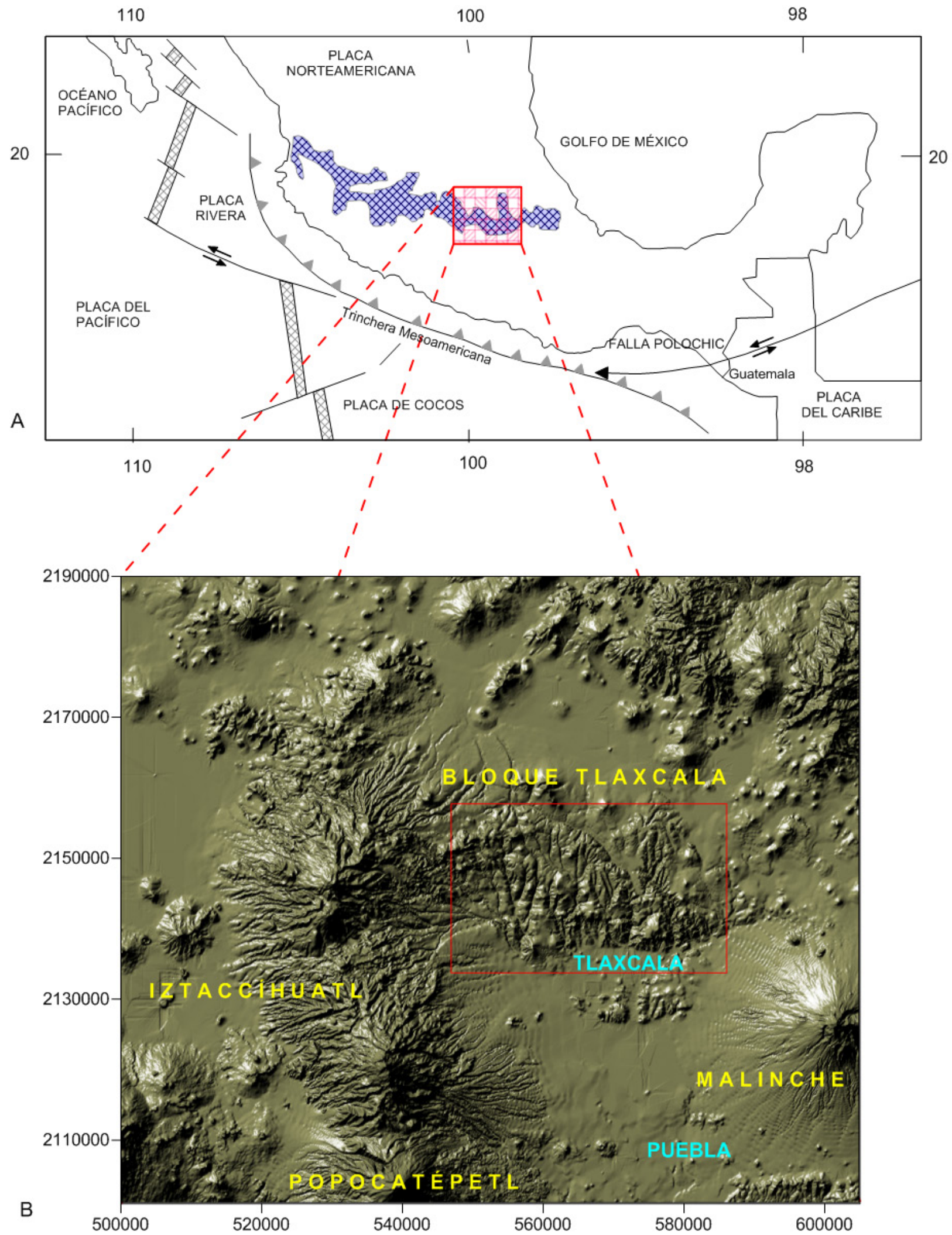

Figura 1. Localización del área de estudio. A) Características tectónicas de México y la ubicación de la Faja Volcánica Transmexicana (modificado de Castro-Govea y Siebe, 2007); B) Bloque Tlaxcala. 
presentan diferentes unidades de paleosuelos que cubren el Cuaternario tardío en Tlaxcala; la relación pedoestratigráfica de cada sección se estableció con base en los fechamientos de ${ }^{14} \mathrm{C}$ (Tabla 1).

La designación de las subunidades se hizo tomando en cuenta la nomenclatura previamente establecida por Sedov et al. (2009) para la Unidad Gris de las secciones Tlalpan S y Mamut: TX1, TX1a, TX1b y TX2 (Figura 2), las cuales fueron reconocidas en las secciones aquí estudiadas. Estas cuatro unidades se relacionan con cuatro periodos específicos: la Etapa Isotópica de Oxígeno 3 (EIO3), el Último Máximo Glacial (UMG), el Glacial tardío y terminal (GT), Holoceno temprano (HT).

De esta manera, TX2 cubre el periodo correspondiente a la EIO3, con edades que oscilan de 30189 a 51798 años cal AP (Tabla 1). La unidad vinculada a la EIO2 es TX1b, con edades que van de los 19663 a 31138 años cal AP (Tabla 1). Cabe mencionar que la fecha de 31138 años cal AP fue tomada de una muestra del horizonte $\mathrm{Bt}$, el cual representa una edad temprana del suelo, en tanto que la de 19663 años cal AP proviene del horizonte AE, que es la edad mínima. Por lo tanto, se considera que TX1b se forma a finales de la EIO3 y continua su desarrollo durante la EIO2, incluyendo el tiempo durante el UMG que comprende el intervalo entre 26500 y 19000 años cal AP (Clark et al., 2009). Las edades de TX1a, que corresponden al GT, son más antiguas que las encontradas en la unidad anterior, a pesar de que sobreyace a TX1b, lo que se explica por "reprocesamiento" de suelos pre-existentes mediante la erosión y su redepositación. Finalmente, TX1 corresponde al HT, con edades de 10426 \pm 88 y $11186 \pm 35$ años cal AP (Tabla 1). Estas edades coinciden con las reportadas por Borejsza y Frederick (2010) para suelos cumúlicos encontrados en la barranca Tenexac, aproximadamente a $30 \mathrm{~km}$ hacia el noroeste del área de estudio.

La figura 2 muestra la correlación estratigráfica entre estas unidades en las diferentes secciones estudiadas, así como con las analizadas por Sedov et al. (2009).

\subsection{Caracterización de las unidades}

\subsubsection{TX2- Etapa Isotópica de Oxígeno 3 (EIO3)}

Los suelos de la unidad TX2, formada durante la EIO3, se identificaron en dos secciones: en Tlalpan C (cima) y en Huexoyucan (base). En el perfil Concepción C esta unidad no fue encontrada, en su lugar se tiene sedimentos coluvio-aluviales.

La unidad TX2 se caracteriza por un fuerte desarrollo de estructura vértica (bloques angulares, muy duros) y una alta acumulación de humus obscuro; son comunes los rasgos reductomórficos como moteados y concentraciones ferruginosas. En cada sección, estos suelos muestran variaciones debido a su ubicación en el relieve. En Tlalpan C, por ejemplo, esta unidad está conformada por dos horizontes: el primero con textura limosa, color pardogrisáceo y pocos recubrimientos de arcilla y humus que sugieren un horizonte de eluviación (6E); a diferencia, el segundo horizonte (6Btg), es más arcilloso, con rasgos de iluviación de arcilla, mayor contenido de materia orgánica, pseudomiscelios en todas direcciones y fuertes

Tabla 1. Fechamientos por radiocarbono de las unidades pedoestratigráficas

\begin{tabular}{|c|c|c|c|c|c|c|c|}
\hline Unidad & Horizonte & Sección & $\begin{array}{l}\text { Material } \\
\text { fechado }\end{array}$ & $\begin{array}{l}\text { No. muestra } \\
\text { Laboratorio }\end{array}$ & $\begin{array}{c}\text { Edad } \\
\left({ }^{14} \mathrm{C} \text { años AP }\right)\end{array}$ & $\begin{array}{c}\text { Edad Calibrada } \\
\qquad(2 \sigma) \\
\text { Años AP }\end{array}$ & $\begin{array}{c}\text { Edad calibrada } \\
\qquad(2 \sigma) \\
\text { Años calendáricos } \\
\text { AC } \\
\end{array}$ \\
\hline \multicolumn{8}{|c|}{ Holoceno temprano } \\
\hline TX1 & $\mathrm{Btg}$ & Concepción C & Humus & Beta250973 & $9,750 \pm 50$ & $11,270-11,180$ & $9,320-9,230$ \\
\hline TX1 & $\mathrm{AC}$ & Huexoyucan & Humus & Beta250974 & $9,260 \pm 50$ & $10,710-10,490$ & $8,760-8,540$ \\
\hline \multicolumn{8}{|c|}{ Etapa Isotópica de Oxígeno 2- Glacial tardío } \\
\hline TX1a* & Agh & Mamut & Humus & $\begin{array}{l}\text { Beta233846 } \\
\text { IGAN-2342 }\end{array}$ & $\begin{array}{c}21,340 \pm 110 \\
24,690 \pm 2,550\end{array}$ & $\begin{array}{l}25,809-25,141^{* *} \\
31,580-26,195 * *\end{array}$ & $\begin{array}{l}23,859-23,191^{* *} \\
29,630-24,250^{* *}\end{array}$ \\
\hline \multicolumn{8}{|c|}{ Etapa Isotópica de Oxígeno 2- Último Máximo Glacial } \\
\hline TX1b* & $\mathrm{AE}$ & Mamut & Humus & $\begin{array}{l}\text { Beta233847 } \\
\text { IGAN-2377 }\end{array}$ & $\begin{array}{c}16,820 \pm 70 \\
17,310 \pm 920\end{array}$ & $\begin{array}{l}20,321-19,763^{* *} \\
21,925-19,663^{* *}\end{array}$ & $\begin{array}{c}18,218-17,882^{* *} \\
19,975-17,713^{* *}\end{array}$ \\
\hline TX1b* & $\mathrm{Bt}$ & Mamut & Humus & $\begin{array}{c}\text { Beta233848 } \\
\text { Hv-24875 }\end{array}$ & $\begin{array}{l}22,070 \pm 120 \\
25,375 \pm 800\end{array}$ & $\begin{array}{l}26,860-26,133^{* *} \\
31,138-29,356^{* *}\end{array}$ & $\begin{array}{l}24,051-23,568 \\
29,188-27,406\end{array}$ \\
\hline \multicolumn{8}{|c|}{ Etapa Isotópica de Oxígeno 3} \\
\hline $\mathrm{TX} 2 *$ & $\mathrm{AB}$ & Mamut & Humus & $\begin{array}{l}\text { IGAN-2376 } \\
\text { Hv-24872 }\end{array}$ & $\begin{array}{l}27,940 \pm 2,400 \\
28,925 \pm 1,750\end{array}$ & $\begin{array}{l}34,727-30,189^{* *} \\
35,031-31,927^{* *}\end{array}$ & -- \\
\hline $\mathrm{TX} 2 *$ & $\mathrm{AB}$ & Tlalpan & Humus & $\begin{array}{l}\text { IGAN-2341 } \\
\text { Hv-24565 }\end{array}$ & $\begin{array}{c}38,160 \pm 5880 \\
>33595\end{array}$ & $\begin{array}{c}48,306-36418^{* *} \\
--\end{array}$ & -- \\
\hline TX2 & $\mathrm{ACo}$ & Huexoyucan & Humus & Beta250975 & $46,320 \pm 870$ & $51,798-47,650 * *$ & -- \\
\hline
\end{tabular}

*Fechamientos reportados por Sedov et al., 2009

** Programa de calibración: CALPAL online http://www.calpal-online.de 


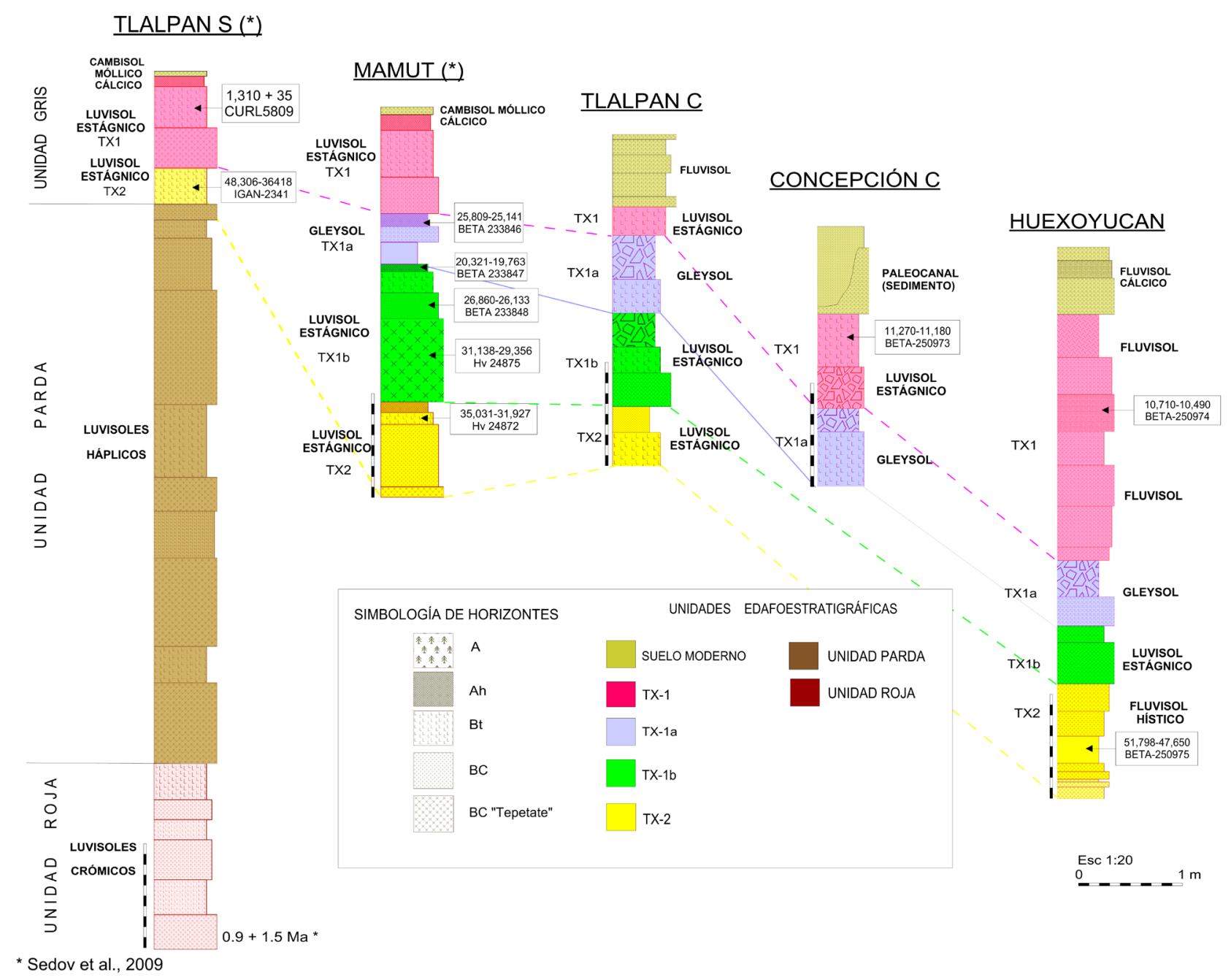

Figura 2. Fechamientos y correlación pedoestratigráfica de los perfiles Tlalpan C, La Concepción C y Huexoyucan. Las edades de los paleosuelos son no calibradas y se reportan en años antes del presente.

rasgos vérticos. La micromorfología evidencia pocos rasgos de intemperismo de los minerales primarios (Figura $3 \mathrm{~A}$ ), a pesar de contener cutanes de arcilla (Figura 3B) y abundantes cutanes de estrés. Cierta cantidad de la arcilla iluviada se encuentra incorporada parcialmente a la matriz.

En la sección Barranca Huexoyucan (ubicada en el área aluvial), TX2 constituye un pedocomplejo con tres paleosuelos $(6 \mathrm{~A} / 6 \mathrm{Ag} / 6 \mathrm{ACo} / 7 \mathrm{Ag} / 7 \mathrm{BC} / 8 \mathrm{AC} / 8 \mathrm{BC} / 8 \mathrm{C})$. El horizonte $6 \mathrm{~A}$ muestra acumulación de humus, arcilla y material orgánico detrítico, mientras que $6 \mathrm{Ag}$ posee rasgos reductomórficos con una serie de laminaciones de óxidos de hierro que siguen la misma configuración que la geoforma. En las secciones delgadas de los horizontes orgánicos se observan acumulaciones de espículas de esponja, diatomeas (especies Eunotia y Pinnularia) y fitolitos, los cuales están incorporados a la matriz (Figura 3C). El material orgánico está separado en forma de láminas, entre las que se intercalan capas delgadas de óxidos de hierro, que le dan una coloración rojiza a la matriz (Figura 3D). Los horizontes de la base muestran un aumento en la cantidad de materia orgánica, evidenciado por la coloración parda oscura de la matriz (Figura 3E); los rellenos de arcilla son densos, completos y laminados, pero deformados (Figura 3F).

Los resultados de los análisis de laboratorio muestran diferencias entre las secciones Tlalpan C y Huexoyucan. En el primer perfil (cima de la toposecuencia) los porcentajes de arcilla varían entre $32.21 \%$ y $51.89 \%$, el carbono orgánico y los valores de susceptibilidad magnética son bajos (Tabla 2). En Huexoyucan el porcentaje de arcilla es más variable, oscilando desde $16 \%$ hasta $88 \%$, lo que refleja su carácter aluvial; mientras que los valores de carbono orgánico también son heterogéneos. De hecho, es en los horizontes $6 \mathrm{Ag}$ y $6 \mathrm{ACo}$ donde se tienen los máximos de carbono orgánico (5\%) de todos los suelos estudiados (Tabla 2). En contraste, la susceptibilidad magnética muestra mínimos en estos horizontes enriquecidos con materia orgánica (Figura 4).

En el paleosuelo orgánico de la sección Huexoyucan 

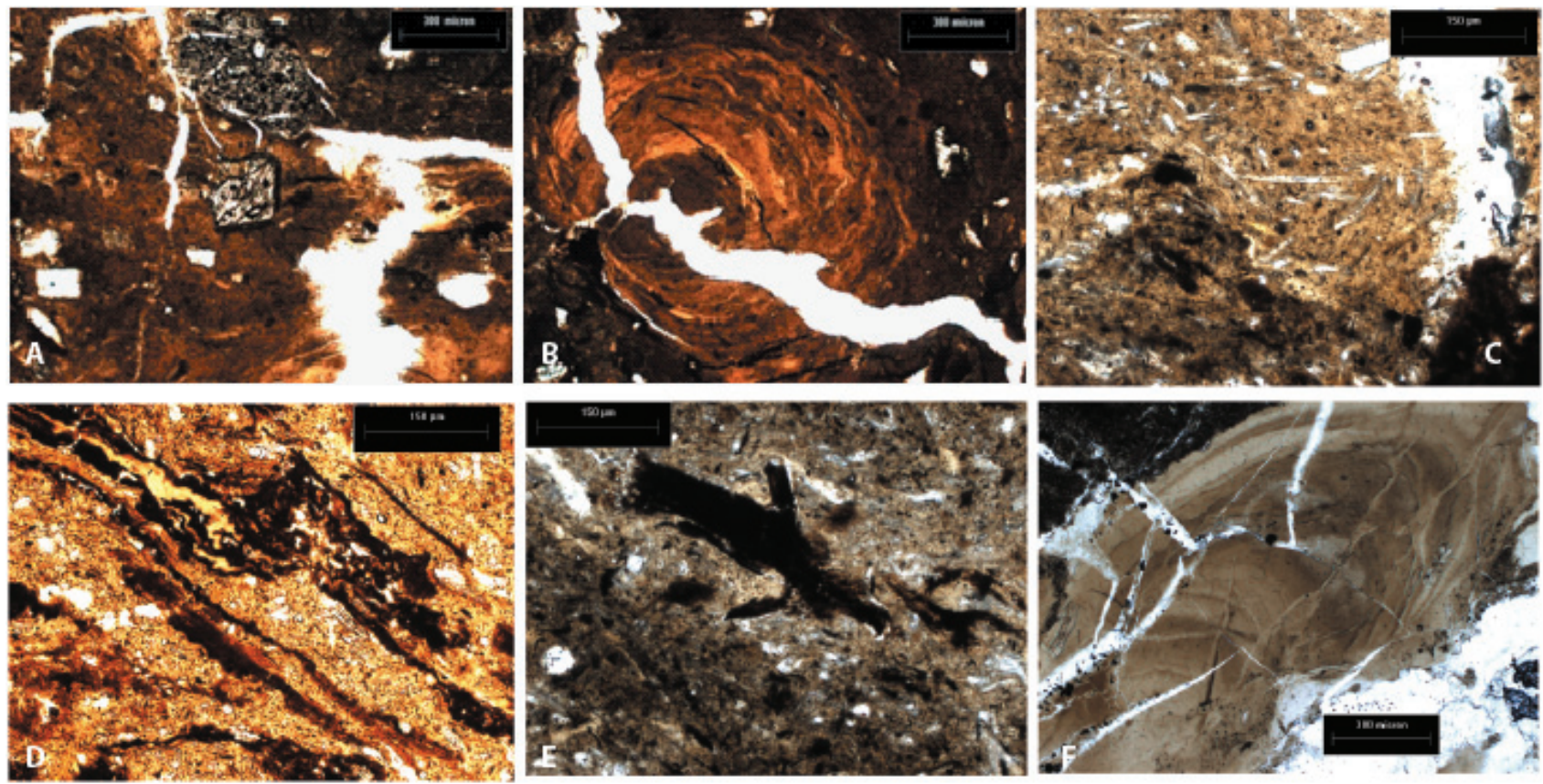

Figura 3. Rasgos micromorfológicos de la unidad pedoestratigráfica TX2 en las secciones Tlalpan C y Huexoyucan. A) revestimientos de arcilla, limo y arena fina con minerales poco intemperizados e incorporados a la matriz, perfil Tlalpan C; B) Cutanes de arcilla deformados en el horizonte 6Btg del perfil Tlalpan C; C) Fitolitos en una matriz impregnada de materia orgánica, horizonte 6Ag del perfil Huexoyucan; D) Laminaciones de material orgánico poco alterado en una matriz de coloración rojiza, en el horizonte 6Ag del Perfil Huexoyucan, E) Restos de de tejido vegetal con poca evidencia de descomposición, horizonte 6ACo del perfil Huexoyucan; F) Relleno denso y laminado de arcilla del horizonte 6 $\mathrm{Ag}_{2}$ del perfil Huexoyucan. Todas bajo luz polarizada.

se realizaron estudios paleobotánicos (Tabla 3), siendo el horizonte $6 \mathrm{Ag}$ el que contiene la mayor variedad de esporas (Polypodium, Triletes y Compuestas) y algas (Botryococcus, Zygnema, Debarya y Spirogyra) con poca concentración de especies terrestres. En la parte superior del horizonte 6ACo las especies terrestres se encuentran mejor representadas y en mayor porcentaje, con especies de Pinus, Quercus, Lycopodium y Polipodium, Amaranthaceae; las especies acuáticas ubicadas en menor porcentaje son algas (Zygnema y Botryococcus). Finalmente, en la parte inferior de 6ACo, nuevamente las especies terrestres son las predominantes. A diferencia de la parte superior en donde los pinos son los más diversos en especies y cantidad, Quercus y Cyperaceas son más abundantes. Asimismo, se registraron diferentes especies de diatomeas como Aulacoseira, Pinnularia y Crysophytas y gran cantidad de espículas de esponja.

Con respecto a los valores de $\delta^{13} \mathrm{C}$ (Tabla 2), las firmas más negativas corresponden a los horizontes $7 \mathrm{Ag}$ y $6 \mathrm{ACo}$ del perfil Huexoyucan (-26.82\% y -26.52\%), aumentando en los horizontes que les sobreyacen, 6A y 6Ag (Tabla 2), los cuales son similares a los encontrados para Tlalpan C $(-22.04 \%)$.

Los suelos que constituyen TX2 en las áreas más drenadas, tanto en Tlalpan C como en las secciones estudiadas por Sedov et al. (2009), Tlalpan S y Mamut, se han clasificado como Luvisoles estágnicos. En Huexoyucan, al fondo del valle, el pedocomplejo se ha clasificado como un Fluvisol hístico (Figura 2).

\subsubsection{TX1b - Etapa Isotópica de Oxígeno 2 (EIO2), Último Máximo Glacial (UMG)}

Los suelos que se desarrollaron durante el Último Máximo Glacial (UMG) se distinguen por un fuerte desarrollo de estructura y porosidad biogénica, mayor iluviación de arcilla y gleyzación. Sin embargo, en la intensidad de tales procesos existen variaciones que dependen de la ubicación de las secciones.

En Tlalpan C (cima), el paleosuelo del UMG (TX1b) está formado por los horizontes $5 \mathrm{Bg} / 5 \mathrm{Btg} / 5 \mathrm{BC}$, mostrando propiedades reductomórficas, en forma de nódulos, moteados y recubrimientos de colores claros en una matriz oscura. Es de llamar la atención la presencia de krotovinas en la parte superior del paleosuelo, en el límite de 5Bg con la unidad que le sobreyace. La mayor acumulación de arcilla se da en 5Btg (Figura 5A); los argilanes son de mayor espesor y recubren tanto las superficies de los agregados como los bioporos (Figura 5A), a diferencia del horizonte 5BC que tiene una textura limo-arenosa con estructura columnar, muestra bioporos sin una dirección preferencial y su matriz es muy compacta (Figura 5B).

En la Barranca Huexoyucan (valle), TX1b (5Ag/5C) tiene una estructura en bloques subangulares; los rasgos reductomórficos predominan, con una alta acumulación de humus y arcilla tanto en la matriz como en la superficie de los agregados, formados por procesos de iluviación (Figura 5C); el horizonte 5C muestra ligeros rasgos de pedogénesis, su textura es limosa con una densidad aparente baja debido al origen volcánico del material (ceniza). Las secciones 
Tabla 2. Propiedades físicas y químicas selectas de las unidades.

\begin{tabular}{|c|c|c|c|c|c|c|}
\hline Horizonte & \multicolumn{3}{|c|}{ Textura } & $\operatorname{COT}(\%)$ & $\begin{array}{c}\text { Isótopos estables } \\
\text { de Carbono }\end{array}$ & $\begin{array}{c}\text { Unidad } \\
\text { Pedoestratigráfica } \\
\end{array}$ \\
\hline \multicolumn{7}{|l|}{ Tlalpan C } \\
\hline Ap & 44.9 & 30.9 & 24.3 & 1.576 & & Suelo moderno \\
\hline $\mathrm{AC}$ & 30.1 & 36 & 33.9 & 0.328 & & Fluvisol \\
\hline $\mathrm{C} 1$ & 44.1 & 33.5 & 22.4 & 0.23 & & \\
\hline $\mathrm{C} 2$ & 18.5 & 32.6 & 48.9 & 0.599 & & \\
\hline $2 \mathrm{AC}$ & 48.1 & 38.7 & 13.2 & 0.992 & -17.18 & \\
\hline $3 \mathrm{Btg}{ }_{1}$ & 20.4 & 30.4 & 49.2 & 0.903 & -18.98 & TX1 \\
\hline $3 \mathrm{Btg}_{2}$ & 12 & 32.5 & 55.5 & 0.401 & & Luvisol estágnico \\
\hline $4 \mathrm{Btg}$ & 8.75 & 38.3 & 52.9 & 0.258 & -18.31 & TX1a \\
\hline $4 \mathrm{Bg}$ & 14.9 & 43.9 & 41.2 & 0.135 & -18.85 & Gleysol \\
\hline $5 \mathrm{Bg} 1$ & 13.1 & 44 & 42.9 & 0.139 & & TX1b \\
\hline $5 \mathrm{Bg} 2$ & 20.7 & 49.9 & 29.4 & 0.175 & & Luvisol estágnico \\
\hline $5 \mathrm{Btg}$ & 22.5 & 46 & 31.5 & 0.159 & -20.82 & \\
\hline $5 \mathrm{BC}$ & 22.8 & 50.1 & 27.1 & 0.213 & & \\
\hline $6 \mathrm{E}$ & 29 & 38.8 & 32.2 & 0.144 & & $\mathrm{TX} 2$ \\
\hline $6 \mathrm{Btg}$ & 14 & 34.1 & 51.9 & 0.19 & -22.04 & Luvisol Estágnico \\
\hline \multicolumn{7}{|c|}{ Concepción C } \\
\hline Paleocanal & 23.6 & 33.6 & 42.8 & 1.117 & & \multirow[t]{2}{*}{ Paleocanal/Sedimento } \\
\hline $\mathrm{ABt}$ & 9.93 & 49.3 & 40.8 & 0.174 & & \\
\hline $2 \mathrm{Bg}$ & 15.4 & 44.3 & 40.2 & 0.575 & -20.54 & TX1 \\
\hline 2Btg & 14.2 & 40.4 & 45.3 & 0.991 & -20.64 & Luvisol Estágnico \\
\hline $3 \mathrm{Bg}$ & 30.4 & 44.5 & 25.1 & 0.113 & -22.22 & TX1a \\
\hline $3 \mathrm{Btg}_{1}$ & 23.3 & 45.5 & 31.2 & 0.134 & & Gleysol \\
\hline $3 \mathrm{Btg}_{2}$ & 22 & 43.6 & 35.4 & 0.142 & & \\
\hline
\end{tabular}

\section{Huexoyucan}

\begin{tabular}{|c|c|c|c|c|c|c|}
\hline A & 73.3 & 15.4 & 11.3 & 1.275 & -17.97 & \multirow{4}{*}{ Fluvisol Cálcico } \\
\hline $\mathrm{AC}$ & 47.6 & 35.6 & 16.8 & 0.731 & & \\
\hline $\mathrm{C}_{1}$ & 67 & 17.7 & 15.2 & 0.405 & & \\
\hline $\mathrm{C}_{2}$ & 19.8 & 51.4 & 28.7 & 1.25 & & \\
\hline $2 \mathrm{AB}$ & 15.1 & 39.6 & 45.3 & 1.046 & -20.89 & TX1 \\
\hline $2 \mathrm{C}_{1}$ & 23.5 & 40.5 & 36 & 0.934 & & Fluvisol \\
\hline $2 \mathrm{C}_{2}$ & 53.9 & 22.3 & 23.8 & 0.477 & & \\
\hline $3 \mathrm{AC}$ & 25 & 31 & 44 & 0.524 & -18.79 & \\
\hline $3 \mathrm{BC}_{1}$ & 39.4 & 35.8 & 24.9 & 0.579 & & \\
\hline $3 \mathrm{BC}_{2}$ & 68.3 & 17.4 & 14.3 & 0.169 & & \\
\hline $3 \mathrm{C}$ & 91.5 & 3.85 & 4.66 & 0.103 & & \\
\hline $4 \mathrm{Bg}$ & 40 & 29.9 & 30.2 & 0.158 & -20.05 & TX1a \\
\hline $4 \mathrm{C}$ & 58.1 & 22.6 & 19.3 & 0.151 & & Gleysol \\
\hline $5 \mathrm{Ag}$ & 12.5 & 28.3 & 59.2 & 0.313 & -18.77 & TX1b \\
\hline $5 \mathrm{C}$ & 31.1 & 61.6 & 7.35 & 0.07 & & Luvisol estágnico \\
\hline $6 \mathrm{~A}$ & 4.14 & 42.6 & 53.2 & 0.765 & -21.26 & TX2 \\
\hline $6 \mathrm{Ag}$ & 0.93 & 11.1 & 88 & 1.865 & -23.86 & Fluvisol hístico \\
\hline $6 \mathrm{ACo}_{1}$ & & & & 5.272 & -26.52 & \\
\hline $6 \mathrm{ACo}_{2}$ & & & & 4.593 & -26.82 & \\
\hline $6 \mathrm{Ag}_{2}$ & 26.7 & 13.2 & 60.2 & 1.105 & -23.19 & \\
\hline $6 \mathrm{BCg}$ & 68.5 & 15.4 & 16.1 & 0.212 & & \\
\hline $7 \mathrm{AC}$ & 27.1 & 32.7 & 40.2 & 0.288 & -10.91 & \\
\hline $7 \mathrm{BC}$ & 57 & 25.6 & 17.4 & 0.178 & & \\
\hline $7 \mathrm{C}$ & 30.8 & 35.4 & 33.7 & 0.189 & & \\
\hline
\end{tabular}

Las unidades de la misma coloración corresponden al mismo nivel pedoestratigráfico y coinciden con los presentados en la Figura 2.

delgadas muestran revestimientos de arcilla en los canales y en la superficie de los microagregados que conforman una microestructura en bloques angulares, el color de la matriz es pardo-obscuro, con una alta porosidad formada por canales delgados (Figura 5D). La fracción mineral está fuertemente intemperizada. Los minerales (piroxenos y anfíboles) presentan una pérdida de más del $50 \%$ del material original (Figura 5E).

En Tlalpan C, se presenta un dominio de la fracción limo (45\% a $50 \%$ ) con valores bajos de susceptibilidad magnética (Figura 4). En Huexoyucan el horizonte 5C es limoso, pero en 5Ag hay mayor proporción de arcilla (59\%) y carbono orgánico $(0.31 \%)$. Los valores de $\delta^{13} \mathrm{C}$ obtenidos para TX1b son más altos que los encontrados en TX2, variando de acuerdo con la posición en el relieve de $-20.82 \%$, en la cima, a $-18.77 \%$, en la base (Tabla 2).

Los suelos de TX1b se clasifican como Luvisoles estágnicos tanto en las secciones de las áreas bien drenadas como Mamut (Sedov et al., 2009) y Tlalpan C, como en Huexoyucan (Figura 2).

\subsubsection{TX1a- Etapa Isotópica de Oxígeno 2 (EIO2), Glacial tardio y terminal (GT)}

Los paleosuelos que se desarrollaron durante el Glacial tardío son reconocibles fácilmente en campo por un cambio en la coloración (gris claro) que evidencia un decremento en la acumulación de humus y contenido de arcilla. Los rasgos reductomórficos son abundantes, variando en forma y tamaño, con una morfología de nódulos, moteados y recubrimientos.

A nivel morfológico pueden observarse algunas diferencias en la toposecuencia. En Tlalpan C (cima) TX1a está formado por los horizontes $4 \mathrm{Btg} / 4 \mathrm{Bg}$, cuyo rasgo característico es la presencia de krotovinas de aproximadamente $30 \mathrm{~cm}$ de diámetro. En Concepción $\mathrm{C}$ (localizado en el área de mayor pendiente), el perfil también muestra estos dos horizontes ( $3 \mathrm{Bg} / 3 \mathrm{Btg})$. En ellos, la superficie de los agregados posee revestimientos arcillosos y manchones con fábrica dendrítica. Las krotovinas también son abundantes, y alcanzan diámetros de hasta $50 \mathrm{~cm}$. En el perfil Huexoyucan $(4 \mathrm{Bg} / 4 \mathrm{C})$ los rasgos reductomórficos son abundantes (moteados y nódulos) con recubrimientos de color pardo-verdoso.

En las propiedades micromorfológicas de las láminas delgadas se observa una matriz de color pardo-claro; los rasgos de gleyzación son frecuentes con concreciones ferruginosas y moteados de hierro en la matriz (Figura 6A); se tienen cutanes de arcilla asociados a bioporos y fracturas (Figura 6B). También se muestran revestimientos entre los bloques angulares que conforman la matriz que bajo luz polarizada presenta fuerte birrefringencia (Figura 6C); la fracción mineral no tiene fuertes rasgos de intemperismo. En Huexoyucan se observan tejidos vegetales parcialmente descompuestos y restos fecales de la actividad biológica. Asimismo, se muestran pápulas y diatomeas deformadas de la especie Pinnularia y Navicula así como fitolitos (Figura 6D).

Los suelos de las tres secciones son limo-arcillosos. Tlalpan C presenta la mayor proporción de arcilla (53\%), pero dada su similitud con la encontrada en el horizonte $3 \mathrm{Btg}_{2}$, perteneciente a TX1, no se descarta el aporte de arcilla iluviada desde este último. La susceptibilidad magnética es baja en todos los horizontes, aunque similar a la encontrada en los otros paleosuelos (Figura 4). Los valores $\delta^{13} \mathrm{C}$ varían de $-18.3 \%$ en los paleosuelos de la cima a $-20.05 \%$ en la base, aunque en Concepción C, en la zona del talud, muestran los valores más bajos (-22-22 
TLALPAN C

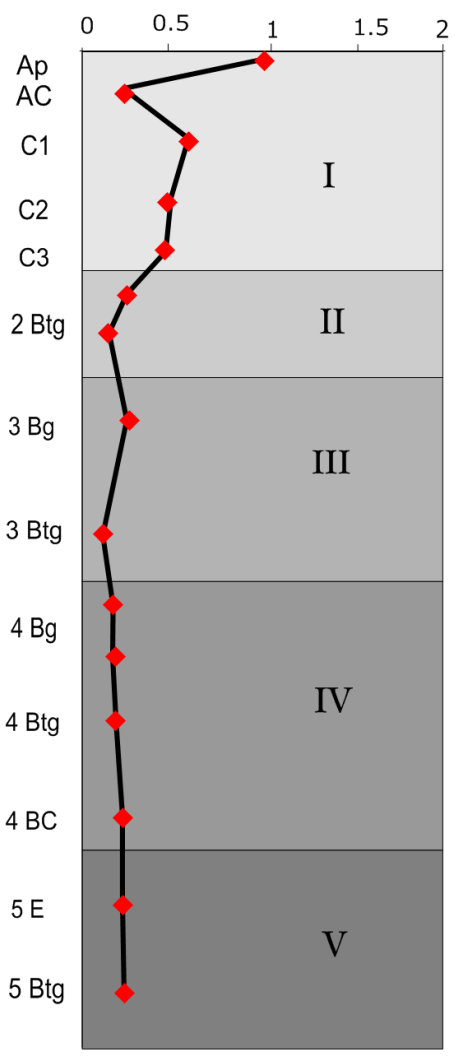

LA CONCEPCIÓN C

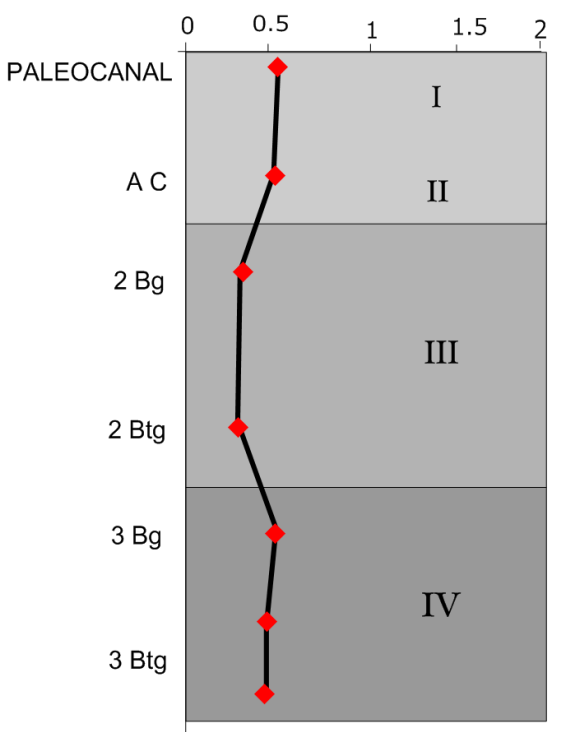

UNIDADES EDAFOESTRATIGRÁFICAS

SUELO MODERNO $(1,310+35$ a. A. P. $)$

TX-1 $(9,750+50$ a. A. P. $)$

TX-1a $(24,690+2550$ a. A. P. $)$

$\operatorname{TX}-1 b(25,525+1180$ a. A. P. $)$

TX-2 (46, $320+870$ a. A. P.)
HUEXOYUCAN

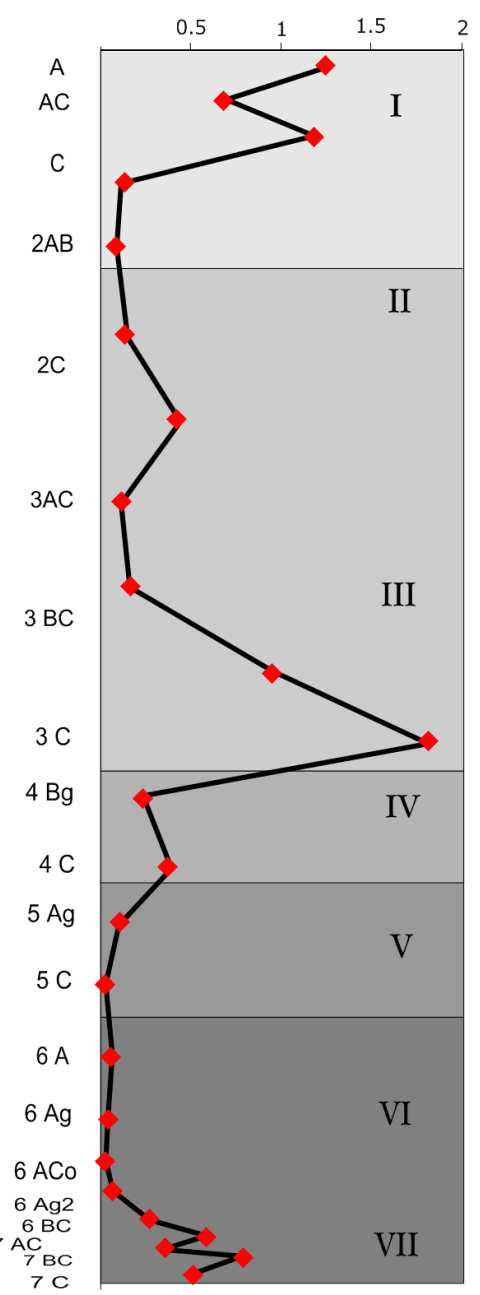

Figura 4. Patrones de susceptibilidad magnética de los perfiles Tlalpan C (cima), Concepción C (talud) y Huexoyucan (fondo del valle).

\%o) (Tabla 2).

Los suelos de TX1a son clasificados como Gleysoles, los cuales se reconocen en todas las posiciones geomorfológicas (Figura 2).

\subsubsection{TX1- Holoceno temprano (HT)}

Los paleosuelos del Holoceno temprano se caracterizan por constituir perfiles con propiedades reductomórficas y alta proporción de arcilla. La cima (Tlalpan C) muestra un solo horizonte, $3 \mathrm{Btg}$ (subdividido en $3 \mathrm{Btg}_{1}$ y $3 \mathrm{Btg}_{2}$ ), con una estructura bien desarrollada en bloques subangulares y rasgos reductomórficos como: concreciones de hierro y manganeso. En la sección Concepción C, TX1 posee los horizontes $2 \mathrm{Bg} / 2 \mathrm{Btg}$, sepultados por un depósito de canal que corta la secuencia. Los horizontes tienen revestimientos arcillosos de color obscuro en las superficies de los agregados, cuya matriz es muy friable. Con la profundidad, la estructura se vuelve de tipo columnar y se incrementa la cantidad de arcilla en la matriz; de hecho, los revestimientos de arcilla tienen un mayor espesor e incluso se observan en bioporos. En el área aluvial, Huexoyucan, se desarrolla un pedocomplejo con dos paleosuelos, cuya configuración es $2 \mathrm{AB} / 2 \mathrm{C} / 3 \mathrm{AC} / 3 \mathrm{BC} / 3 \mathrm{C}$; el primero es un suelo muy profundo, más desarrollado y más compacto que el superior. Se caracterizan por tener una textura limoarcillosa, estructura en bloques subangulares y canales biogénicos dentro de los agregados. Los recubrimientos obscuros localizados en la superficie de los agregados disminuyen gradualmente con la profundidad. En el segundo, aumenta la cantidad de materia orgánica y muestra una estructura más desarrollada, asimismo se incrementan los rasgos de iluviación de arcilla que se observan como halos amarillentos rodeando los bioporos y concreciones ferruginosas.

La micromorfología muestra que TX1 posee una microestructura más compacta y con mayor contenido de materia orgánica; las concreciones de hierro se observan redondeadas y revestidas de arcilla, con un límite abrupto 
Tabla 3. Conteos de polen y diatomeas en los horizontes orgánicos que se localizan en la base de la sección pedoestratigráfica Huexoyucan. Los resultados que se reportan son en número de granos por cada especie y horizonte.

\begin{tabular}{|c|c|c|c|c|}
\hline \multirow{2}{*}{ Especie } & \multicolumn{3}{|c|}{ Horizonte } & \multirow{2}{*}{ Ambiente } \\
\hline & 6Agh & 6ACo (sup) & 6ACO (inf) & \\
\hline \multicolumn{5}{|l|}{ POLEN } \\
\hline Pinus spp. & 16 & 13 & 23 & \multirow{4}{*}{ Bosque } \\
\hline Alnus spp. & 3 & 1 & 2 & \\
\hline Quercus spp. & 12 & 6 & 8 & \\
\hline Poaceae & 9 & 8 & 5 & \\
\hline Amaranthaceae & 7 & 9 & 13 & \multirow{10}{*}{ Pantano } \\
\hline Cheno-Amaranthaceae & 7 & 9 & 13 & \\
\hline Compositae & 6 & 7 & 3 & \\
\hline Cyperaceae & 5 & 4 & 11 & \\
\hline Botryococcus & 14 & 7 & 4 & \\
\hline Zygnemathaceae & 6 & 4 & 2 & \\
\hline Debarya & 7 & 2 & 3 & \\
\hline Spirogyra & 5 & 4 & 1 & \\
\hline Polypodium & 1 & 2 & 3 & \\
\hline Triletes & 2 & 1 & 5 & \\
\hline \multicolumn{5}{|l|}{ DIATOMEAS } \\
\hline Navicul a spp. & 2 & 1 & 6 & Agua \\
\hline Hantszchia spp. & 3 & 1 & 7 & dulces, \\
\hline Gomphonema spp. & 6 & 3 & 11 & neutras o \\
\hline Cymbella spp. & 8 & 3 & 15 & muy poco \\
\hline Aulacoseira spp. & 11 & 6 & 22 & $\begin{array}{c}\text { ácida } \\
\text { Pantano }\end{array}$ \\
\hline Epithemia turgida & 2 & 1 & 5 & $\begin{array}{c}\text { alcalino } \\
\text { poco }\end{array}$ \\
\hline Epithemia spp. & 7 & 5 & 12 & profundo \\
\hline Eunotia papilo & 2 & 2 & 4 & Pantano \\
\hline Eunotia spp. & 9 & 6 & 13 & ácido \\
\hline Crysophyta & 4 & 2 & 9 & $\begin{array}{c}\text { Agua de } \\
\text { baja }\end{array}$ \\
\hline Spicula esponja & 25 & 18 & 45 & $\begin{array}{c}\text { temperatura } \\
\text { Agua }\end{array}$ \\
\hline Pinnularia spp. & 12 & 7 & 28 & $\begin{array}{c}\text { profunda en } \\
\text { turbera } \\
\text { Niveles }\end{array}$ \\
\hline Gemosclera & 5 & 2 & 9 & $\begin{array}{c}\text { bajos del } \\
\text { cuerpo de } \\
\text { agua }\end{array}$ \\
\hline
\end{tabular}

con la matriz (Figura 7A). Los revestimientos de arcilla se observan limpios pero con coloraciones rojizas por enriquecimiento con hierro (Figura 7B), la matriz presenta fisuras, fragmentos de tejido vegetal descompuesto (Figura 7C) y fragmentos de roca (Figura 7D). En su límite con el suelo moderno se observan laminaciones.

Las secciones delgadas de los paleosuelos desarrollados en la ladera (perfil Concepción C) muestran minerales poco intemperizados (Figura 7E). En algunos horizontes se observan diatomeas (Figura 7F) deformadas e incorporadas a la matriz (especie Pinnularia); los cutanes de arcilla son más gruesos y laminados (Figura 7G), también impregnados con materia orgánica. Por su parte, los suelos del fondo del valle (perfil Huexoyucan) se muestran recubrimientos de goethita sobre los cutanes que recubren los bioporos (Figura $7 \mathrm{H}$ ).

TX1 en Tlalpan C tiene $55.54 \%$ de arcilla disminuyendo hacia los paleosuelos en una posición topográfica más baja, con valores de arcilla de $40 \%$ en Concepción C. En
Huexoyucan, los porcentajes de arcilla al igual que los valores de susceptibilidad magnética son más heterogéneos, variando desde $5 \%$ hasta $43 \%$ (Tabla 2) y de $0.08 \mu \mathrm{m}^{3} / \mathrm{kg}$ a $1.75 \mu \mathrm{m}^{3} / \mathrm{kg}$ (Figura 4).

Los valores $\delta^{13} \mathrm{C}$ son más similares en Tlalpan C (-18.98 $\%$ ) y Huexoyucan (-18.79\%), si bien en este último punto, el paleosuelo más joven ( $2 \mathrm{AB})$, tiene un valor más elevado $(-20.89 \%)$. En Concepción C el valor es menor y similar a este último (Tabla 2).

TX1 se ha clasificado como Luvisol estágnico en las secciones de Tlalpan S y Mamut (Sedov et al., 2009) así como en Tlalpan C y Concepción C. En Huexoyucan, esta unidad es un Fluvisol (Figura 2).

\subsubsection{Suelo moderno (Holoceno tardio)}

El suelo moderno constituye la cubierta del Holoceno tardío. En la figura 2 se puede observar que este último está caracterizado por ser un Cambisol móllico cálcico en las posiciones más elevadas (Tlalpan S) que cambia a suelos de naturaleza coluvio-aluvial, con varios horizontes A sepultados, los cuales fueron encontrados en las partes bajas de las Barrancas Tlalpan S y el Mamut (Sedov et al., 2009); en Concepción C, el suelo moderno está erosionado, por lo que sólo se reconoce un depósito aluvial con una débil pedogénesis.

\section{Discusión}

\subsection{Historia paleoambiental}

Las propiedades que exhiben los paleosuelos estudiados en la región de Tlaxcala permiten establecer la historia paleoambiental desde la EIO3 hasta el Reciente y compararla con los datos publicados para el centro de México.

\subsubsection{Etapa Isotópica de Oxígeno 3 (EIO3)}

En el perfil Huexoyucan (base de la pendiente) se desarrollaron horizontes orgánicos en condiciones anóxicas, así como horizontes con fuerte acumulación de humus obscuro, iluviación y traslocación de material orgánico, sugiriendo un cuerpo de agua somero (pantanoso) con vegetación de helechos (esporas Polypodium y Triletes) $\mathrm{y}$ taxa de humedal (algas). El registro de las diatomeas de este depósito acuático muestra una marcada estacionalidad: incremento de agua superficial durante los periodos húmedos y aguas ligeramente turbias en las estaciones secas.

En Tlalpan C, la formación de un Luvisol estágnico, la presencia de rasgos de iluviación y, particularmente, la acumulación de humus obscuro y arcilla en los horizontes B, permiten interpretar su formación bajo condiciones húmedas y frías, las cuales promueven la traslocación de la arcilla y la materia orgánica en conjunto. Los valores de $\delta^{13} \mathrm{C}(-22 \%)$ evidencia la presencia de vegetación predominantemente de tipo C3. La analogía de los suelos modernos (formados en zonas de bosque mixto bajo un clima frío y húmedo) con 

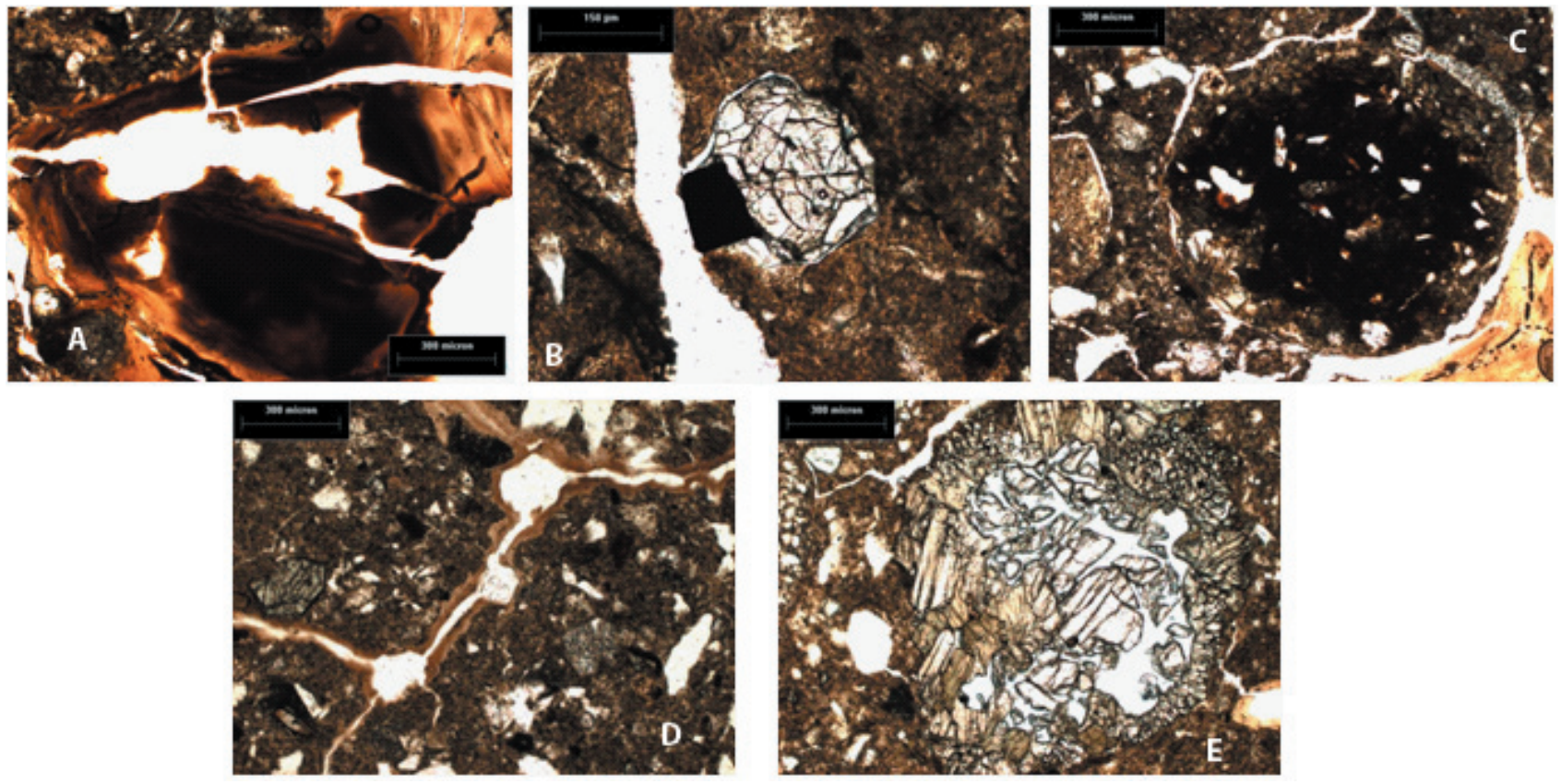

Figura 5. Rasgos micromorfológicos de la unidad pedoestratigráfica TX1b en las secciones Tlalpan C y Huexoyucan (luz natural). A) Revestimientos laminados de arcilla y hierro, horizonte 5Btg de la unidad en Tlalpan C; B) Matriz compacta del horizonte 5BC con coloraciones pardo obscuras. C) Nódulo de hierro con límites abruptos con la matriz en el horizonte 5Ag; D) Microestructura en bloques angulares limitados por canales delgados con revestimientos de arcilla; E) Piroxeno intemperizado, mostrando pérdida del material original. Todas bajo luz natural.
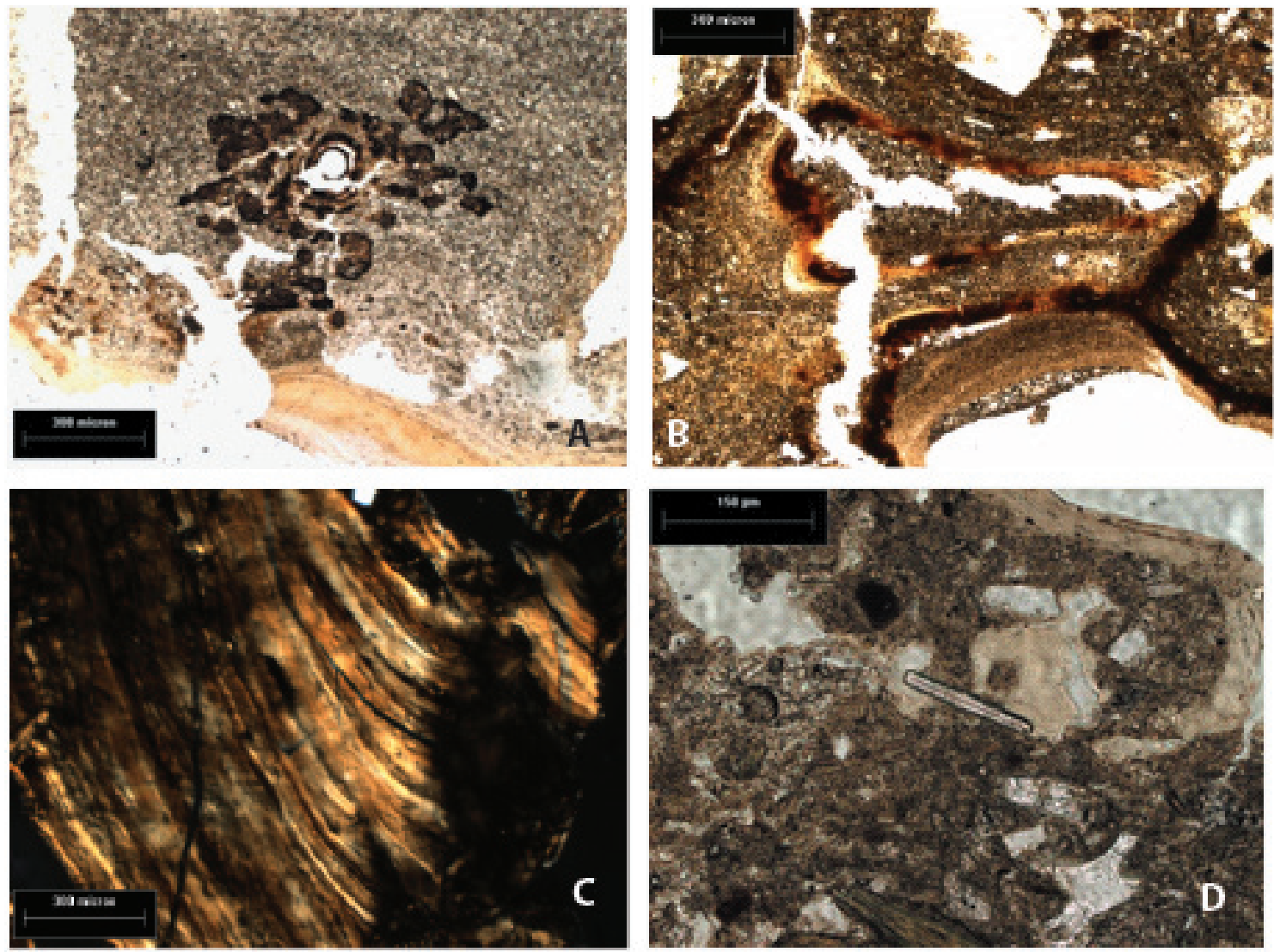

Figura 6. Rasgos micromorfológicos de la unidad pedoestratigráfica TX1a en las secciones Tlalpan C, La Concepción C y Huexoyucan (luz natural). A) Concentraciones de hierro en el horizonte $3 \mathrm{Bg}$ en Concepción C, B) Revestimientos de arcilla laminados y fracturados en el horizonte 4Bg del perfil Huexoyucan; C) Cutanes de arcilla bajo luz polarizada del horizonte 4Btg del perfil Tlalpan C; D) Diatomeas de la especie pinularia en el horizonte 4Bg del perfil Tlalpan C. 

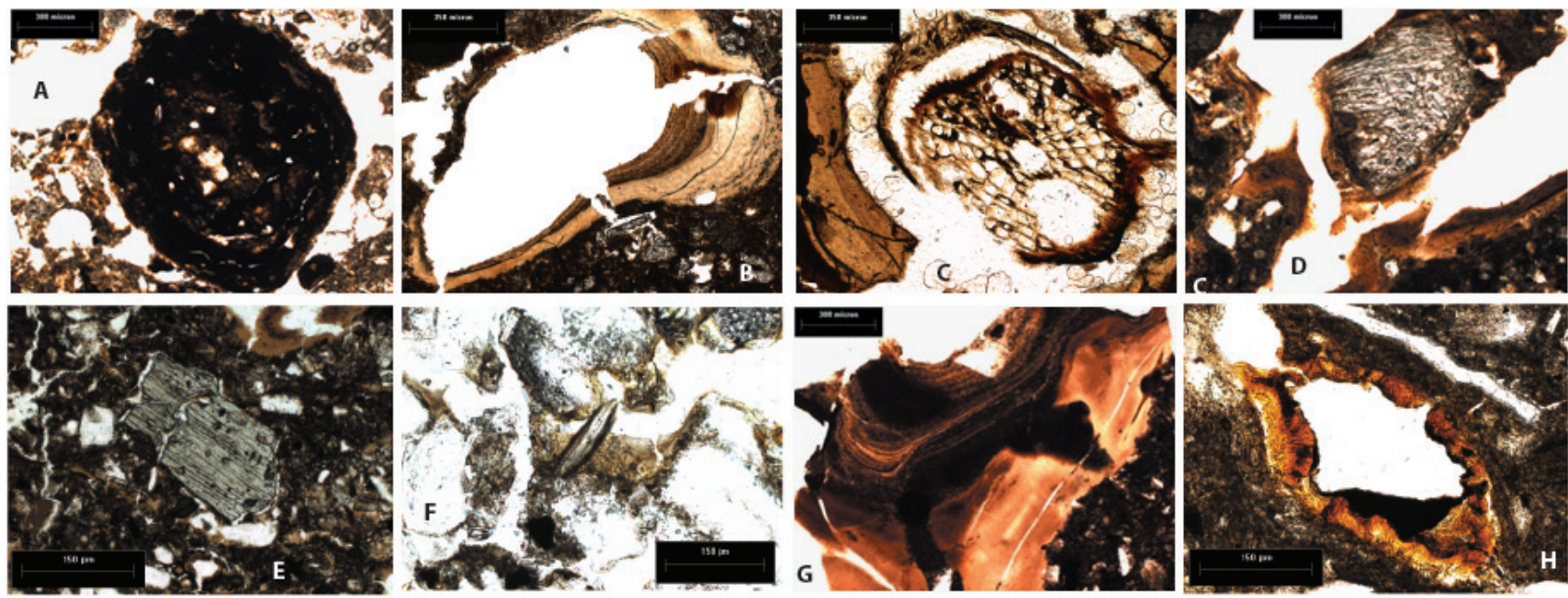

Figura 7. Rasgos micromorfológicos de la unidad pedoestratigráfica TX1 en las secciones Tlalpan C, La Concepción C y Huexoyucan (luz natural). A) Concreciones de hierro redondeadas en el horizonte 2Btg del perfil Tlalpan C; B) Cutanes de arcilla con enriquecimiento de hierro en el horizonte 2Btg del perfil Tlalpan C; C) Tejido vegetal en el horizonte 3AC del perfil Huexoyucan, D) Fragmento de roca en el horizonte 2Bg del perfil La Concepción C, E) Anfíbol con alteración pelicular en el horizonte 2Btg del perfil La Concepción C, F) Diatomea de la especie pinularia en la matriz del horizonte $3 \mathrm{AC}$ del perfil Huexoyucan, G) Cutanes gruesos y laminados, enriquecidos con materia orgánica y hierro en el horizonte 2Btg del perfil la Concepción $\mathrm{C}$; y H) Recubrimientos de arcilla y goetita en el horizonte $2 \mathrm{AB}$ del perfil Huexoyucan.

TX2, en Tlalpan C, indica una paleovegetación forestal, lo que se corrobora con los análisis paleobotánicos (Tabla 3), los cuales también muestran la asociación de Pinus, Quercus y Alnus, cohabitando con pastos (ChenopodiaceaeAmaranthacea) y vegetación no arbórea (Poaceae) y acuática (Cyperaceae). Sedov et al. (2009) consideran que la formación de la unidad TX2 ocurrió en ambientes con una temperatura media anual de $7-8^{\circ}$, que es menor a la actual en el Bloque Tlaxcala. Sin embargo, los mismos autores consideran que estas condiciones no fueron constantes ya que los paleosuelos en Tlalpan S y Mamut muestran rasgos vérticos, signo de una marcada estacionalidad en la precipitación. Los rasgos vérticos en Tlalpan C también son congruentes con esta aseveración. En Huexoyucan, los resultados obtenidos apoyan esta idea, ya que los cambios en el cuerpo de agua son evidentes dada la fluctuación en los valores de tamaño de grano, susceptibilidad magnética, carbono orgánico y, sobre todo, en los valores de $\delta^{13} \mathrm{C}$ que van de $-20.91 \%$ a $-26.82 \%$, estacionándose, hacia el final de su desarrollo, en -21.2 \%o. Este valor se asocia con condiciones más secas, o de mayor temperatura que promovieron que el cuerpo de agua se secara.

En resumen, el conjunto de propiedades analizadas en TX2 permiten inferir condiciones fías y húmedas en la zona (Figura 8A), las cuales se interpretan con base en las siguientes observaciones:

- Alta concentración de materia orgánica con buen grado de humificación.

- Fuertes procesos de gleyzación, presentes en ambas posiciones topográficas. En Tlalpan C, ubicado en la cima, estas características son notables, lo que permite inferir una alta humedad en el ambiente, y no sólo producto del ascenso del manto freático en las partes más bajas (fondo del valle).

- Valores $\delta^{13} \mathrm{C}$ entre -23 \%o y -21\%o documentan la presencia dominante de vegetación tipo $\mathrm{C} 3$.

- Taxa mesofíticas y de humedal como las algas (Botryococcus, Zygnema, Debarya y Spirogyra).

- Diatomeas de la especie Eunotia, Pinnularia, Cymbella y Navicula refieren un clima húmedo con periodos estacionales marcados.

- Diatomeas de Chrysophyta y Hantszchia, sugieren un ambiente frío, con temperaturas entre 5 y $12^{\circ} \mathrm{C}$ (Patrick y Reimer, 1966, 1975).

- Polen arbóreo de Pinus, Quercus y Alnus.

El registro glacial en el centro de México es escaso, debido a la falta de una cronología precisa (Vázquez-Selem y Heine, 2004), aunque en La Malinche está documentado un avance, el del glaciar MI, con una edad de 36000-32000 años AP (Heine, 1975, 1994), que refleja condiciones frías para la región. En el Nevado de Toluca, el registro de paleosuelos también evidencian condiciones frías y húmedas, con fases secas, durante el intervalo que va de 50000 a 37000 años AP (Sedov et al., 2003). En la región del Valle de Teotihuacán, Solleiro-Rebolledo et al. (2011) reportan la presencia de paleosuelos formados en ambientes húmedos durante la EIO3, bajo un ecosistema forestaltemplado (Sycheva et al., 2010), con fases de mayor sequía que propiciaron el desarrollo de rasgos vérticos.

\subsection{2. Último Máximo Glacial (UMG)}

Las evidencias paleopedológicas del Último Máximo Glacial (UMG), que se basan en las observaciones hechas en los perfiles estudiados en Tlalpan C y Huexoyucan, ambos con Luvisoles estágnicos (Unidad TX1b, edad de 20042 a 30247 años cal AP), sugieren una humedad permanente 

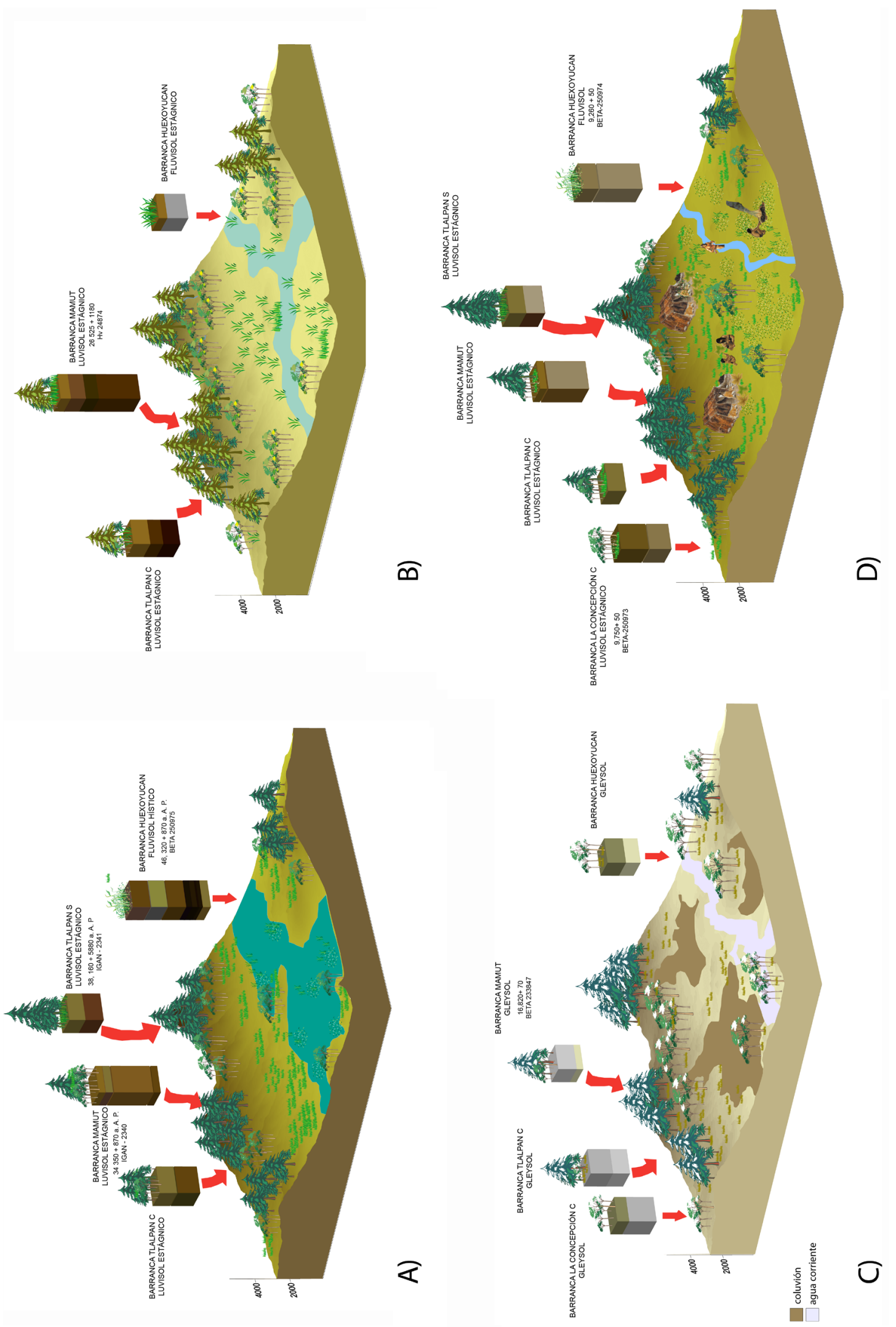

Figura 8. Reconstrucción hipotética de las condiciones ambientales en las Etapas Isotópicas de Oxígeno 3 y 2. Las características y distribución de los rasgos del paisaje fueron deducidas con base en los análisis físicos, químicos y morfológicos de los paleosuelos del Bloque Tlaxcala. A) Reconstrucción de la paleotoposecuencia durante la EIO3, unidad pedoestratigráfica TX2; B) Paleotoposecuencia de la EIO2, Último Máximo Glacial, unidad pedoestratigráfica TX1b; C) Paleotoposecuencia de la EIO2, Glacial Tardío, unidad TX1a; D) Paleotoposecuencia del Holoceno Temprano, unidad pedoestratigráfica TX1, en la cual se infiere ocurrió el poblamiento temprano. 
alternando con ciertas fases secas. Sin embargo, estas fases secas sólo se interpretan a partir del registro de isótopos estables de carbono, cuyas firmas dan valores más altos: $-20.81 \%$ y $-18.77 \%$. Por ello se infiere que estas fases secas fueron breves y sólo tuvieron impacto en la vegetación y no afectaron al conjunto de propiedades desarrolladas durante las fases húmedas.

El perfil Tlalpan $\mathrm{C}$, que representa condiciones ambientales en una altitud mayor (cima), muestra propiedades como alto contenido de limo y arcilla, estructura en bloques angulares, acumulación de arcilla en bioporos y en las caras de los agregados, todo lo cual se asocia a una vegetación forestal. Asimismo, presenta fuertes rasgos estágnicos, a pesar de su posición elevada. Los valores de $\delta^{13} \mathrm{C}$, que fluctúan entre $-20.81 \%$ y -18.77 $\%$, sugieren una vegetación abierta con árboles y pastos, que dan una firma combinada entre plantas C3 y C4. El arreglo de la estructura a nivel micromorfológico que señala la actividad biogénica observada en las secciones delgadas (ocasionada por las raíces) corrobora la presencia de los pastizales (Figura 8B).

En Huexoyucan (base de la pendiente) se desarrolla un Fluvisol con fuertes propiedades estágnicas (concreciones de hierro y manganeso moteados amarillentos) con un valor de $\delta^{13} \mathrm{C}$ de $-18.8 \%$ también se relacionan con una vegetación mixta.

Por lo tanto, las condiciones de alta humedad no son producto de la posición en el relieve, ya que la acumulación de arcilla, los revestimientos de la misma y los rasgos reductomórficos se presentan en la cima de la pendiente (Tlalpan C). De acuerdo con los resultados de Sedov et al. (2009) en la Barranca Mamut, TX1b muestra la mayor cantidad de arcilla iluviada e intemperismo de los minerales, ambos rasgos observados en secciones delgadas, lo que documenta ambientes de mayor humedad, posiblemente relacionado con una disminución de temperatura. La Figura 8B ilustra el paisaje propuesto para este periodo.

Comparando con otros registros paleoecológicos, Ohngemach y Straka (1983) señalan que la Cuenca PueblaTlaxcala entre los 31000 y 18000 años AP se caracterizó por tener un clima frío y relativamente seco intercalado con periodos cortos más fríos y húmedos. Para este periodo Heine (1975) encuentra un suelo denominado $\mathrm{fBo}_{1}$ en las faldas de La Malinche, el cual es un Andosol profundo cuyas propiedades reflejan condiciones húmedas (Sedov et al., 2009).

Discrepa el registro glacial del centro de México (Vázquez-Selem y Heine, 2004), el cual evidencia un clima seco y frío, con avances en el volcán Ajusco: El Marqués entre los 27000 y 26000 años AP, Santo Tomás entre los 25000 y 24000 años AP.

Lauer (1979) documenta un clima extremadamente frío y húmedo entre 21000 y 13000 años AP favoreciendo la formación del suelo $\mathrm{fBo}_{1}$ (Andosol) y el avance del glaciar MI en el volcán La Malinche, el cual presenta fluctuaciones en temperatura y precipitación. También en el volcán
Iztaccíhuatl se registra el avance glaciar Hueyatlaco I entre los 20000 y 17500 años AP y Hueyatlaco II entre los 17000 y 14000 años AP (Lachniet y Vázquez-Selem, 2005). En Cofre de Perote también se presenta un avance glaciar que corresponde con el Hueyatlaco I (Lachniet y VázquezSelem, 2005), en tanto que en el volcán Ajusco el glaciar El Albergue avanza entre los 21000 y 16000 años AP (White y Valastro, 1984).

Por otro lado, los registros lacustres de la Cuenca de México, específicamente los paleolimnológicos, indican que durante el Último Máximo Glacial (26000 a 18000 años AP) el clima tiende a condiciones frías y secas, con una baja diversidad de vegetación forestal y taxa subacuática como Isöetes y algas (Lozano-García y Xelhuanzti-Lopez, 1997; Metcalfe et al., 2000). Contrariamente, los datos polínicos de la sección pedoestratigráfica Tepexpan, evidencian condiciones templadas y húmedas alrededor de 20000 y 27000 años AP (Sedov et al., 2010). Bradbury (1989) también documenta un clima frío y húmedo, aunque la ausencia de avances glaciares en las montañas altas, son interpretadas a la vez, con condiciones áridas. En los paleosuelos del Nevado de Toluca, integrados en la unidad pedoestratigráfica PT2 (Sedov et al., 2001; 2003) encuentran propiedades relacionadas con climas húmedos con fases temporales secas.

\subsubsection{Glacial tardio-terminal}

El Glacial tardío y terminal está representado por la unidad pedoestratigráfica TX1a, constituida por Gleysoles, tanto en Tlalpan C como Huexoyucan (Figura 2 y Tabla 2), que muestran un desarrollo débil, controlado por procesos pedogenéticos rápidos: gleyzación y acumulación de humus, ambos con tiempos característicos de formación de decenas a cientos de años (Targulian y Krasilnikov, 2007). De esta forma, su presencia en diferentes partes de la toposecuencia estudiada, se interpretan como producto de periodos cortos de pedogénesis y estabilidad del paisaje, intercalados con periodos de mayor duración en la sedimentación y la erosión, en un ecosistema frío y húmedo (Figura $8 \mathrm{C}$ ). La inversión en las edades encontradas apoya esta idea, ya que TX1a resultó ser más antiguo que TX1b (Tabla 1), a pesar de su posición estratigráfica. Dicha inversión indica redepositación de material de suelo (posiblemente erosionado de TX1b). Estos procesos de erosión y sedimentación se producen durante periodos de inestabilidad geomórfica intensa en el GT, interrumpidos por periodos cortos de estabilización, registrados en las fases de formación de los Gleysoles. Heine (1994) detecta dos periodos de inestabilidad geomórfica a finales del Pleistoceno, alrededor de 16.5 a $12 \mathrm{ka}$ AP y entre 10 y $8 \mathrm{ka}$ AP, relacionado con los avances glaciares MII y MIII, respectivamente, como consecuencia del enfriamiento que se produjo por la descarga de agua glacial en el Golfo de México, desde el Atlántico norte, al fundirse las masas de hielo del glaciar Lauréntida. Las fases de estabilidad de estos periodos, se considera, fueron favorables para la formación de los paleosuelos $\mathrm{fBo}_{2}$ y $\mathrm{fBo}_{3}$, los cuales corresponden con 
TX1a y TX1, respectivamente. De hecho, los procesos de gleyzación y la acumulación de humus oscuro en las caras de los peds, detectados en TX1a, se han interpretado como resultado de condiciones frías en un ecosistema forestal, coincidiendo con lo propuesto por coincidiendo con lo propuesto por Sedov et al., 2009.

Los registros paleolimnológicos del GT en la cuenca de México (16000 - 12000 años cal AP) evidencian niveles lacustres altos en Chalco, con diatomeas de agua dulce, somera y polen de vegetación boscosa, abierta que va cambiando a comunidades más cerradas hacia el Glacial terminal (Caballero et al., 2010). En Texcoco y Tecocomulco se documentan condiciones áridas interpretadas por la presencia de hiatos en las secuencias a los 14500 y 15000 años cal AP (Caballero et al., 1999; 2010). La interpretación integral del registro paleolimnológico señala que en el Glacial terminal se da un incremento de temperatura de 2 ${ }^{\circ} \mathrm{C}$, lo cual se refleja en la expansión del bosque (Caballero et al., 2010).

Vázquez-Selem y Heine (2004) señalan que en esta época, se favorece el desarrollo de morrenas recesionales entre 14000 y 13000 años cal AP, seguido por el retraimiento de los glaciares entre 13000 - 12000 años cal AP, con un avance alrededor de los 12000 años cal AP. Sin embargo, los mencionados autores proponen que, dada la falta de avances glaciares durante el Younger Dryas (época fría a nivel global), efectivamente fue fría, pero muy seca.

Sin embargo, los registros paleopedológicos en el Valle de Teotihuacán, al igual que lo encontrado en Tlaxcala, coinciden en la interpretación de condiciones de clima húmedo y frío, el cual permitió el desarrollo de un Luvisol estágnico (13066 años cal AP) en el perfil Maseca (Solleiro-Rebolledo et al., 2006). En el Glacis de Buenavista, Morelos, destaca la asociación de Albeluvisol y Gleysoles (15820-14070 años cal AP), cuya presencia actual en el mundo ocurre en zonas con clima boreal o templado bajo bosque de coníferas con inviernos fríos y veranos cortos y frescos (Solleiro-Rebolledo et al., 2003; Díaz-Ortega et al., 2010). Se destaca que en ambas zonas, Valle de Teotihuacan y Glacis de Buenavista, los paleosuelos mencionados están cubiertos por depósitos relacionados con sedimentos aluviales y lahares, respectivamente (Solleiro-Rebolledo et al., 2011; Díaz-Ortega et al., 2010), relacionados con eventos catastróficos producidos por periodos de inestabilidad geomórfica, coincidiendo con lo encontrado en Tlaxcala.

\subsubsection{Holoceno temprano (HT)}

La unidad pedoestratigráfica TX1 (edad 11270 - 10490 años cal AP) evidencia las condiciones paleoambientales predominantes en el Holoceno Temprano, la cual se conforma por paleosuelos que presentan fuertes propiedades gleycas y reductomórficas. A diferencia de TX1a, en donde se observa una homogeneidad en el tipo de suelos sin importar la posición en el paisaje, los cambios en las propiedades de TX1 sí cambian en función de la geoforma, de tal manera que en la cima tiene mayores rasgos de iluviación, en tanto que en la base, presenta más signos de gleyzación y de procesos sedimentarios, conformando Fluvisoles. Esto sugiere que la humedad en el ambiente es menor, con temperaturas bajas (dada la acumulación de humus oscuro en la matriz del suelo), con una vegetación de tipo mixto $\mathrm{C} 3 / \mathrm{C} 4$, dados los valores de las firmas isotópicas de $-18 \%$ a $-21 \%$ (Figura $8 D$ ).

El registro glacial coincide de alguna manera, ya que se tienen dos avances glaciales en el Iztaccíhuatl, el Milpulco I entre los 12000 y 10500 años cal AP; y el Milpulco II entre los 8300 y 7300 años cal AP; y uno en el volcán La Malinche entre los 10000 y 9000 años cal AP (MIII); (Vázquez-Selem y Heine, 2004), que señalan la disminución de las temperaturas.

Por su parte, los registros paleolimnológicos del lago de Chalco indican un incremento en la precipitación durante el Holoceno temprano (Lozano-García y Ortega-Guerrero, 1998; Metcalfe et al., 2000). En Tepexpan, en el límite del lago de Texcoco, el registro muestra una clara tendencia a condiciones más secas, ya que a los 11615 años cal AP se presenta un pedosedimento formado en un ecosistema pantanoso, en tanto que hacia el Holoceno medio y tardío, los suelos poseen carbonatos secundarios en la matriz, que marcan condiciones de mayor aridez (Sedov et al., 2010).

En la región de Tlaxcala, para este periodo se ha registrado la activación de los procesos de inestabilidad en el paisaje (10200 años cal AP), evidenciados en el incremento en la descarga de los ríos y en la sedimentación aluvial, provocando incisiones geomorfológicas en el valle, como consecuencia de la transición de condiciones glaciales a condiciones interglaciales (Borejsza y Frederick, 2010). Reflejo de tales condiciones de inestabilidad se tiene en la presencia de suelos cumúlicos en varias de las barrancas de Tlaxcala y, en particular en Concepción C, en donde estos suelos son sepultados por gruesos depósitos aluviales.

\subsection{Condiciones ambientales durante el poblamiento temprano}

De particular interés son las condiciones ambientales durante el poblamiento temprano de la región. Dentro de los trabajos efectuados en los sitios bien documentados como Peñón, Tlapacoya, Texcal e inclusive Valsequillo, la evidencia se centra en las poblaciones y sus patrones de dispersión, más que en las condiciones paleoambientales. Algunos aspectos se han documentado como el registro de ambientes con alternancia de climas húmedos y secos pero con predominio de climas más secos y fríos que el actual (González-Quintero, 1986). Dado que las evidencias más claras en el centro de México, se enmarcan en los 11000 años no calibrados AP (González et al., 2003), es decir en el Glacial terminal-Holoceno que, como se ha mencionado, es un periodo con cambios drásticos en el ambiente, de fuerte inestabilidad geomorfológica, en el que se presentan condiciones alternantes de estabilidad e inestabilidad del 
paisaje, lo que limita los espacios de poblamiento.

Tomando en cuenta los resultados obtenidos en este trabajo, así como la interpretación de la distribución de los ecosistemas deducida a partir de las propiedades morfológicas, químicas y micromorfológicas de las unidades pedoestratigráficas analizadas, se puede esbozar hipotéticamente, los escenarios en los cuales pudo ocurrir el poblamiento temprano.

En el Glacial terminal, documentado en la unidad TX1a, se ha interpretado un ambiente húmedo y frío, con procesos de inestabilidad geomórfica que causan erosión y depósito de materiales. Estos paisajes pudieron limitar la expansión de los grupos. Sin embargo en el Holoceno temprano, en el que se desarrolla TX1, aunque también se presentan procesos relacionados con la activación de los ríos, las condiciones parecen ser más favorables para el poblamiento. A diferencia de TX1a, en el que es dominante el retrabajamiento de los materiales y la presencia de mayor sedimentación (dinámica más activa en el valle), en TX1 los suelos se desarrollan en ambientes húmedos y menos fríos, que pudieron limitar los cuerpos de agua, en una red aluvial que les permitiera la utilización del agua y de los recursos aledaños en el entorno.

En este escenario, los valles, al contrario de las etapas anteriores, se tienen suelos más drenados, con mayor desarrollo de estructura biogénica, acumulación de humus y presencia de mesofauna. Todo esto documenta claramente un entorno bien drenado, en donde los suelos hidromórficos están más limitados. En las fases anteriores (EIO3, UMG, GT), todos los suelos muestran propiedades gleycas.

En el HT la estructura espacial del paisaje señala la existencia de condiciones de saturación de agua en el fondo del valle en donde, además, también ocurren procesos de sedimentación, en tanto que hacia las laderas y partes más elevadas, los suelos son más estables, con vegetación de pastizal. Este paisaje es propicio para el poblamiento, ya que por un lado ofrece sitios drenados para el asentamiento temporal; cuerpos de agua que abastecen de este recurso; y pastos y agua que atraen animales, favoreciendo la caza (Figura 8D).

Es así que la unidad TX1 representa un elemento estratigráfico importante en la prospección de sitios arqueológicos, relacionados al poblamiento temprano en la zona de Tlaxcala.

\section{Conclusiones}

Las unidades pedoestratigráficas estudiadas permiten esbozar una tendencia de cambio magistral desde la Etapa Isotópica de Oxígeno 3 (EIO3) hasta el Reciente. En primer lugar, los paleosuelos pleistocénicos demuestran propiedades marcadamente diferentes a las observadas en los suelos holocénicos.
Las unidades pedoestratigráficas estudiadas corresponden a cuatro periodos:

TX2 que corresponde a la (EIO3).

TX1b formada a finales de EIO3, cubriendo el Último Máximo Glacial (UMG).

TX1a se asocia con el Glacial tardío y terminal (GT).

TX1 que se formó en el Holoceno temprano (HT).

Estas unidades se estudiaron a lo largo de una toposecuencia (Tlalpan C - cima; Concepción C - talud; Huexoyucan - fondo del valle) de manera que se pudieran observar las modificaciones en sus propiedades en respuesta de los cambios en el relieve y en el cima.

En la EIO3 se desarrollan Luvisoles con rasgos vérticos, los cuales están presentes en las posiciones de mayor altitud, cambiando hacia Fluvisoles hísticos en la base de la toposecuencia, evidenciando un clima con oscilaciones entre condiciones frías-húmedas y secas estacionalmente. Hacia el UMG los Luvisoles se presentan tanto en la cima como en la base, de lo que se infiere el predominio de un clima húmedo y frío más uniforme.

Las características de periodos de inestabilidad geomórfica se observan en GT, por la presencia de suelos de bajo grado de desarrollo que contienen material retrabajado y redepositado, y que conforman Gleysoles en las diferentes posiciones del relieve. Los paleosuelos del HT se han clasificado como Luvisoles con propiedades estágnicas propios de un clima húmedo y frío, pero bien drenados en las partes altas. En el fondo del valle son claros procesos de erosión y sedimentación que reflejan la fuerte actividad geomórfica de la zona. En la prospección arqueológica del poblamiento temprano de la región, TX1 es un marcador estratigráfico que puede facilitar la labor de encontrar materiales de interés.

\section{Agradecimientos}

Este trabajo fue elaborado gracias al financiamiento de los proyectos PAPIIT 1N110107 y PAPIIT IN117709 de la Dra. Elizabeth Solleiro, y del proyecto CONACYT 43746 del Dr. Jorge Gama, a quien se agradece su apoyo en las diferentes fases de esta investigación. Asimismo, agradecemos la colaboración de Kumiko Shimada por las determinaciones de carbono orgánico; a Elena Lounejeva, Pedro Morales, Edith Cienfuegos del Laboratorio de Isótopos Estables del Instituto de Geología por los análisis de isótopos estables de carbono; a Jaime Díaz, Héctor Cabadas y René Alcalá, por el apoyo en los análisis de suelos; a Socorro Lozano, Margarita Caballero y Beatriz Ortega quienes apoyaron la realización de los análisis de polen, diatomeas y susceptibilidad magnética, respectivamente. Finalmente, se agradece al Eligio Jiménez por la preparación de las secciones delgadas. 


\section{Referencias}

Borejsza, A., Rodríguez-López, I., Frederick, C.D., Bateman, M.D., 2008, Agricultural slope management and soil erosion at La Laguna, Tlaxcala, Mexico: Journal of Archaeological Science, 35, 1854-1866.

Borejsza, A., Frederick, C.D., 2010, Fluvial response to Holocene climate change in low-order streams of central Mexico: Journal of Quaternary Science, 25, 762-781.

Bradbury, J.P., 1989, Late Quaternary lacustrine paleoenvironments in the Cuenca de Mexico: Quaternary Science Reviews, 8, 75-100.

Bullock P., Fedoroff, N., Jongerius, A., Stoops, G., Tursina, T., Babel, U., 1985, Handbook for Soil Thin Section Description: Wolverhampton, Reino Unido, Waine Research Publications, $152 \mathrm{p}$.

Caballero, M., Lozano, S., Ortega, B., Urrutia, J., Macías, J.L., 1999, Environmental characteristics of Lake Tecocomulco, northern basin of Mexico, for the last 50000 years: Journal of Paleolimnology, 22, 399-411.

Caballero, M., Lozano-García, S., Vázquez-Selem, L., Ortega, B., 2010, Evidencias de cambio climático y ambiental en registros glaciales y en cuencas lacustres del centro de México durante el último máximo glacial: Boletín de la Sociedad Geológica Mexicana, 62, 359-377.

CalPal Online, 2007, Cologne Radiocarbon \& Paleoclimate Research Package: Colonia, Alemania, University of Cologne, publicado Mayo 2007, disponible en <http://www.calpal-online.de>, consultado 10 de Octubre de 2011.

Castro-Govea R., Siebe, C., 2007, Late Pleistocene-Holocene stratigraphy and radiocarbon dating of La Malinche volcano, Central Mexico: Journal of Volcanology and Geothermal Research, 162, 20-42.

Clark, P.U., Dyke, A.S., Shakun, J.D., Carlson, A.E., Clark, J., Wohlfarth, B., Mitrovica, J.X., Hostetler, S.W., McCabe, A.M., 2009, The Last Glacial Maximum: Science, 325, 710-714.

Díaz-Ortega, J., Solleiro-Rebolledo, E., Sedov, S., Cabadas, H., 2010, Paleosuelos y tepetates del Glacís de Buenavista Morelos (México): testigos de eventos climáticos de la transición Pleistoceno-Holoceno: Boletín de la Sociedad Geológica Mexicana, 62, 469-486.

Fiedel, S.J., 2006, Clovis First: still the best theory of native American origins, en Jiménez-López, J.C., Polaco, O.J., Martínez Sos, G., Hernández Flores, G. (eds.), 20 Simposio Internacional del Hombre Temprano en América: México, Instituto Nacional de Antropología e Historia, 45-60.

Fujita, H., Duarte, M.T., Bate, L.P., 2006, Una probable ocupación desde el Pleistoceno en la Cueva Babisuri, Isla Espíritu Santo, Baja California Sur, México, en Jiménez-López, J.C., Polaco, O.J., Martínez, Sos, G., Hernández Flores, G. (eds.), 20 Simposio Internacional del Hombre Temprano en América: México, Instituto Nacional de Antropología e Historia, 61-72.

García E., 1988, Modificaciones al Sistema de Clasificación Climática de Köppen: México, Offset Larios, 219 p.

González-Quintero L., 1986, Análisis polínicos de los sedimentos, en Lorenzo, J.L., Mirambell, L., (eds.), Tlapacoya: 35000 Años de Historia del lago de Chalco: México, Instituto Nacional de Antropología e Historia, 157-166.

González, S., Jimenez-López, J.C., Hedges, R., Huddart, D., Ohman, J.C., Turner, A., Pompa, Padilla, J.A., 2003, Earliest humans in the Americas: new evidence from México: Journal of Human Evolution, 44, 379-387.

González, S., Huddart, D., Bennett, M.R., González-Huesca, A., 2006, Human footprints in Central Mexico older than 40000 years: Quaternary Science Reviews, 25, 201-222.

Heine, K., 1975, Studien zur jungquartären Glazialmorphologie mexikanischer Vulkane-mit einem Ausblick auf die Klimaentwicklung: Weisbaden, Franz Steiner Verlag, Das MexikoProjekt der Deutschen Forschungsgemeinschaft 7, 178 p.

Heine, K., 1994, The late-glacial moraine sequences in Mexico: is there evidence for the Younger Dryas event?: Palaeogeography, Palaeoclimatology, Palaeoecology, 112, 113-123.
Heine K., 2003, Paleopedological evidence of human-induced environmental change in the Puebla-Tlaxcala area (Mexico) during the last 3500 years: Revista Mexicana de Ciencias Geológicas, 20, $235-244$.

International Union of Soil Sciences (IUSS) Working Group WRB, 2006, World reference base for soil resources. A framework for international classification, correlation and communication: Rome, Food and Agricultural Organization of the United Nations (FAO), World Soil Resources Reports, 103, 132 p.

Klink, H.J., 1973, La división de la vegetación natural en la región PueblaTlaxcala: Proyecto Puebla-Tlaxcala, Fundación Alemana para la Investigación Científica, Comunicaciones, 7, 38-42.

Kozlowski, J.K., Bandi, H.G., 1992, El problema de las raíces asiáticas del primer poblamiento de América: Espacio, Tiempo y Forma, Serie I, Prehistoria y Arqueología, 5, 15-72.

Krammer, K., Lange-Bertalot, H., 1991. Bacillariophyceae 3. Centrales, Fragilariaceae, Eunotiaceae. Sübwasserflora von Mitteleuropa: Stuttgart, Gustav Fisher Verlag, 576 p.

Lachniet, M.S., Vázquez-Selem, L., 2005, Last Glacial Maximum equilibrium line altitudes in the circum-Caribbean (Mexico, Guatemala, Costa Rica, Colombia, and Venezuela): Quaternary International, 138-139, 129-144.

Lauer, W., 1979, Medio ambiente y desarrollo cultural en la región PueblaTlaxcala: Proyecto Puebla-Tlaxcala, Fundación Alemana para la Investigación Científica, Comunicaciones, 16, 29-54.

Lermo-Samaniego, J., Bernal-Esquia, I., 2006, Zonificación sísmica del estado de Tlaxcala, México: Boletín de la Sociedad Geológica Mexicana, 58, 215-221.

Lorenzo, J.L., Mirambell, L., 1986a, Preliminary Report on Archaeological and Paleoenvironmental Studies in the area of El Cedral (San Luis Potosí), México, 1977-1980, en Bryan, A. (ed.), New Evidene for the Pleistocene Peopling of the Americas: Orono, Maine, E.U.A., University of Maine at Orono, Center for the Study of the First Americans, 107-113.

Lorenzo, J.L., Mirambell, L., 1986b, Tlapacoya: 35000 años de historia del Lago de Chalco: México, Instituto Nacional de Antropología e Historia, Colección Científica, 159 p.

Lozano-García, M. del S., Xelhuantzi-Lopez, M.S., 1997, Some problems in the late Quaternary pollen records of Central Mexico: Basins of Mexico and Zacapu: Quaternary International, 43-44, 117-123.

Lozano-García, S., Ortega-Guerrero, B., 1998, Late Quaternary environmental changes of the central part of the Basin of Mexico; correlation between Texcoco and Chalco basins: Review of Palaeobotany and Palynology, 99, 77-93.

Mehra, O.P., Jackson, M.L., 1960, Iron oxide removal from soils and clays by a dithionite-citrate-bicarbonate system buffered with sodium bicarbonate buffer: Clays and Clay Minerals, 7, 317-327.

Metcalfe, S.E., O'Hara, S.L., Caballero, M., Davies, S.J., 2000, Records of Late Pleistocene-Holocene climatic change in Mexico - a review: Quaternary Science Reviews, 19, 699-721.

Mirambell, L., 1978, Tlapacoya: A late Pleistocene site in central Mexico, en Bryan, A.L. (ed.), Early man in America from a circum-Pacific Perspective: Occasional Papers, Department of Anthropology, University of Alberta, 1, 221-222.

Mooser, F., Montiel A., Zuñiga, A., 1996, Nuevo mapa geológico de las Cuencas de México, Toluca y Puebla. Estratigrafía, tectónica regional y aspectos geotérmicos, 1:100000: México, Comisión Federal de Electricidad 1937-1996, Carta Texcoco, 5b, 1 mapa.

Ohngemach, D., Straka, H., 1983, Resumen en Español del trabajo en Puebla de la comunidad alemana, en Lauer, W. (ed.), Das MexicoProjeckt der Deutschen Forschungsgemeinschaft 18: Beiträge zur Vegetation- und Klimageschichte im Bebiet von Puebla - Tlaxcala: Wiesbaden, Hesse, Alemania, Steiner, 143-161.

Patrick, R., Reimer, C.W., 1966, The diatoms of the United States: Academy of Natural Sciences of Philadelphia Monographs, 1, 13, 688 p.

Patrick, R., Reimer, C.W., 1975, The diatoms of the United States: Academy of Natural Sciences of Philadelphia Monographs, 2, 13, 213 p. 
Reimer P.J., Baillie, M.G.L., Bard, E., Bayliss, A., Beck, J.W., Bertrand, C.J.H., Blackwell, P.G., Buck, C.E., Bur, G.S., Cutler, K.B., Damon, P.E., Edwards, R. L., Fairbanks, R.G., Friedrich, M., Guilderson, T.P., Hogg, A.G., Hughen, K.A., Kromer, B., McCormac, G., Manning, S., Bronk Ramsey, C., Reimer, R.W., Remmele, S., Southon, J.R., Stuiver, M., Talamo, S., Taylor, F.W., van der Plicht, J., Weyhenmeyer, C. E., 2004, IntCal04 Terrestrial Radiocarbon Age Calibration, 0-26 cal kyr BP: Radiocarbon, 46, 1029-1049.

Retallack, G.J., 1990, Soils of the past: An introduction to paleopedology: Reino Unido, Blackwell Science, 404 p.

Rico, R., Vilaclara, G., Miranda, J., Cañetas, J., 1997, Origin of laminations in Tlaxcala paleolake, Mexico: International Verein Limnology, 26, 838-841.

Sedov, S., Solleiro-Rebolledo, E., Gama-Castro, J.E., Vallejo-Gómez E., González-Velázquez, A., 2001, Buried paleosols of the Nevado de Toluca: an alterative record of Late Quaternary environmental change in central Mexico: Journal of Quaternary Science, 16, 375-389.

Sedov, S., Solleiro-Rebolledo, E., Morales-Puente, P., Arias-Herreía, A., Vallejo-Gómez, E., Jasso-Castañeda, C., 2003, Mineral and organic components of the buried paleosols of the Nevado de Toluca, Central Mexico as indicators of paleoenvironments and soil evolution: Quaternary International, 106-107, 169-184.

Sedov, S., Solleiro-Rebolledo, E., Terhorst, B., Solé, J., Flores-Delgadillo, M.L., Werner, G., Poetsch, T., 2009, The Tlaxcala basin paleosol sequence: A multiscale proxy of middle to late Quaternary environmental change in central Mexico: Revista Mexicana de Ciencias Geológicas, 26, 448-465.

Sedov, S., Lozano-García, S., Solleiro-Rebolledo, E., McClung de Tapia, E., Ortega-Guerrero, B., Sosa-Nájera, S., 2010, Tepexpan revisited: A multiple proxy of local environmental changes in relation to human occupation from a lake shore section in Central Mexico, Geomorphology, 122, 309-322.

Solleiro-Rebolledo, E., Sedov, S., Gama-Castro, J., Flores-Román, D., Escamilla-Sarabia, G., 2003, Paleosol-sedimentary sequences of the Glacis de Buenavista, Central Mexico: Interaction of Late Quaternary pedogenesis and volcanic sedimentation: Quaternary International, 106-107, 185-201.

Solleiro-Rebolledo, E., Sedov, S., McClung de Tapia, E., Cabadas, H., Gama-Castro, J., Vallejo-Gómez, E., 2006, Spatial variability of environment change in the Teotihuacan Valley during the Late Quaternary: Paleopedological inferences: Quaternary International, 156-157, 13-31.
Solleiro-Rebolledo, E., Sycheva, S., Sedov, S., McClung de Tapia, E., Rivera-Uria, Y., Salcido-Berkovich, C., Kuznetsova, A., 2011, Fluvial processes and paleopedogenesis in the Teotihuacan Valley, México: Responses to late Quaternary environmental changes: Quaternary International, 233, 40-52.

Sycheva, S.A., Sedov, S.N., Solleiro-Rebolledo, E., 2010, Izmeneniya landshaftov dolini r. San-Pablo v konce pozdnego pleistocena (Teotihuacan, Mexico) (Late Pleistocene landscape changes in the San Pablo valley (Teotihuacan, Mexico): Geomorfologia, 3, 90-100 (en ruso).

Targulian, V.O., Goriachkin, S.V., 2004, Soil memory: Types of records, carriers, hierarchy and diversity: Revista Mexicana de Ciencias Geológicas, 21, 1-8.

Targulian, V.O., Krasilnikov, P.V., 2007, Soil system and pedogenic processes: Self-organization, time scales, and environmental significance: Catena, 71, 373-381.

Vázquez-Selem, L., Heine, K., 2004, Late Quaternary glaciation of Mexico, en Ehlers, J., Gibbard, P.L., Hughes, P.D. (eds.), Quaternary Glaciations - Extent and Chronology: Amsterdam, Elsevier, Developments in Quaternary Science, 2, 233-242.

Vilaclara, G., Martinez-Mekler, G., Cuna, E., Ugalde, E., 2010, Diatominferred palaeoenvironmental changes of a Pliocene lake disturbed by volcanic activity: Journal of Paleolimnology, 44, 203-215.

Werner, G., Aeppli, H., Miehlich, G., Schönhals, E. 1978, Los suelos de la cuenca alta de Puebla-Tlaxcala y sus alrededores. Comentarios a un mapa de suelos: Proyecto Puebla-Tlaxcala, Fundación Alemana para la Investigación Científica, Comunicaciones, 6, $95 \mathrm{p}$.

White, S.E., Valastro Jr., S., 1984, Pleistocene glaciation of volcano Ajusco, central Mexico, and comparison with the standard Mexican glacial sequence: Quaternary Research, 21, 21-35.

Manuscrito recibido: Diciembre 12, 2010.

Manuscrito corregido recibido: Noviembre 1, 2011.

Manuscrito aceptado: Diciembre 15, 2011. 